\title{
Toward understanding the formation of multiple systems ${ }^{\star}$ A pilot IRAM-PdBI survey of Class 0 objects
}

\author{
A. J. Maury ${ }^{1}$, Ph. André ${ }^{1}$, P. Hennebelle ${ }^{2}$, F. Motte ${ }^{1}$, D. Stamatellos ${ }^{3}$, M. Bate ${ }^{4}$, A. Belloche ${ }^{5}$, \\ G. Duchêne ${ }^{6,7}$, and A. Whitworth ${ }^{3}$ \\ ${ }^{1}$ Laboratoire AIM, CEA/DSM-CNRS-Université Paris Diderot, IRFU/Service d'Astrophysique, C.E. Saclay, Orme des Merisiers, \\ 91191 Gif-sur-Yvette, France \\ e-mail: amaury@eso.org \\ 2 Laboratoire de radioastronomie, UMR 8112 du CNRS, Ecole normale supérieure et Observatoire de Paris, 24 rue Lhomond, \\ 75231 Paris, France \\ 3 School of Physics \& Astronomy, Cardiff University, Cardiff, CF24 3AA, Wales, UK \\ ${ }^{4}$ School of Physics, University of Exeter, Stocker Road, Exeter EX4 4QL, UK \\ 5 Max-Planck Institut für Radioastronomie, Auf dem Hügel 69, 53121 Bonn, Germany \\ 6 Astronomy Department, University of California, Berkeley, CA 94720-3411, USA \\ 7 Laboratoire d'Astrophysique de Grenoble, Université Joseph Fourier, BP 53, 38041 Grenoble Cedex 9, France
}

Received 16 October 2009 / Accepted 13 January 2010

ABSTRACT

\begin{abstract}
Context. The formation process of binary stars and multiple systems is poorly understood. The multiplicity rate of Class II premain-sequence stars and Class I protostars is well documented and known to be high ( 30\% to 50\% between $\sim 100$ and 4000 AU). However, optical / near-infrared observations of Class I/Class II YSOs barely constrain the pristine properties of multiple systems, since dynamical evolution can quickly alter these properties during the protostellar phase.

Aims. Here, we seek to determine the typical outcome of protostellar collapse and to constrain models of binary formation by core fragmentation during collapse, using high-resolution millimeter continuum imaging of very young (Class 0 ) protostars observed at the beginning of the main accretion phase.

Methods. We carried out a pilot high-resolution study of 5 Class 0 objects, including 3 Taurus sources and 2 Perseus sources, using the most extended (A) configuration of the IRAM Plateau de Bure Interferometer (PdBI) at $1.3 \mathrm{~mm}$. Our PdBI observations have a typical $H P B W$ resolution $\sim 0.3^{\prime \prime}-0.5^{\prime \prime}$ and rms continuum sensitivity $\sim 0.1-1 \mathrm{mJy} / \mathrm{beam}$, which allow us to probe the multiplicity of Class 0 protostars down to separations $a \sim 50 \mathrm{AU}$ and circumstellar mass ratios $q \sim 0.07$.

Results. We detected all 5 primary Class 0 sources in the $1.3 \mathrm{~mm}$ dust continuum. A single component associated with the primary Class 0 object was detected in the case of the three Taurus sources, while robust evidence of secondary components was found toward the two Perseus sources: L1448-C and NGC1333-IR2A. We show that the secondary $1.3 \mathrm{~mm}$ continuum component detected $\sim 600$ AU south-east of L1448-C, at a position angle close to that of the $\mathrm{CO}(2-1)$ jet axis traced by our data, is an outflow feature directly associated with the powerful jet driven by L1448-C. The secondary $1.3 \mathrm{~mm}$ continuum component detected $\sim 1900$ AU southeast of NGC1333-IR2A may either be a genuine protostellar companion or trace the edge of an outflow cavity. Therefore, our PdBI observations revealed only wide (>1500 AU) protobinary systems and/or outflow-generated features.

Conclusions. When combined with previous millimeter interferometric observations of Class 0 protostars, our pilot PdBI study tentatively suggests that the binary fraction in the $\sim 75-1000 \mathrm{AU}$ range increases from the Class 0 to the Class I stage. It also seems to argue against purely hydrodynamic models of binary star formation. We briefly discuss possible alternative scenarios to reconcile the low multiplicity rate of Class 0 protostars on small scales with the higher binary fraction observed at later (e.g. Class I) evolutionary stages.
\end{abstract}

Key words. stars: formation - binaries: general - ISM: clouds - ISM: jets and outflows

\section{Introduction}

Understanding the formation of multiple systems is a major unsolved problem in star formation research (e.g. Tohline 2002). While most solar-type $\left(0.5 \leq M_{\odot} \leq 2 M_{\odot}\right)$ pre-main sequence (PMS) stars are observed to be in binary systems with typical separations 10-300 AU (e.g., Duchêne et al. 2004, 2007, see also below), the detailed manner in which individual prestellar cores fragment (or not) during collapse to form multiple (or

^ Based on observations carried out with the IRAM Plateau de Bure Interferometer. IRAM is supported by INSU/CNRS (France), MPG (Germany), and IGN (Spain). single) stars is still poorly understood and highly debated (see Goodwin et al. 2007 for a review). Even the typical outcome of cloud core collapse is unclear since it has been argued that most stars may actually form as single objects (Lada 2006). The argument is based on the fact that most low-mass stars (with $M_{\star}<0.5 M_{\odot}$ ) are single and that the stellar initial mass function (IMF) is significantly more populated below $0.5 M_{\odot}$ than above (e.g. Kroupa 2001; Chabrier 2005). Despite conventional wisdom, it is therefore conceivable that most low-mass prestellar cores may collapse to single stars.

It is generally believed that multiple systems form by dynamical rotationally-driven fragmentation at the end of (or shortly 
after) the first collapse phase of prestellar cores, when the central $\mathrm{H}_{2}$ density reaches $n_{\text {crit }} \sim 3 \times 10^{10} \mathrm{~cm}^{-3}$ and the equation of state of the gas switches from isothermality to adiabacity (Goodwin et al. 2007). Purely hydrodynamic SPH simulations of rotating cloud core collapse show that a very low level of initial core turbulence (e.g. $E_{\text {turb }} / E_{\text {grav }} \sim 5 \%$ ) leads to the formation of a multiple system (Goodwin et al. 2004; Hennebelle et al. 2004; Commerçon et al. 2008). In such SPH simulations, fragmentation is driven by a combination of rotation/turbulence and occurs in large ( $\gtrsim 100 \mathrm{AU})$ disk-like structures or "circumstellar accretion regions" (CARs - cf. Goodwin et al. 2007). These CARs are not rotationally supported and are highly susceptible to spiral instabilities which fragment them into small- $N$ multiple systems with $N>2$, typically $N \sim 3-4$ components at radii $\leq 150 \mathrm{AU}$ in the equatorial plane (Goodwin et al. 2004; Fromang et al. 2006).

However, a very different outcome is found in simulations of magnetized core collapse, as shown by recent results obtained with MHD codes using both grid techniques (Fromang et al. 2006; Hennebelle \& Teyssier 2008; Machida et al. 2005; Mellon $\&$ Li 2008) and the SPH technique (Price \& Bate 2007). These new MHD simulations indicate that the presence of an even moderate magnetic field strongly modifies angular momentum transport during collapse and at least partly suppresses core fragmentation, often leading to the formation of a single object. Price \& Bate (2007) and Hennebelle \& Teyssier (2008) conclude that binary star formation is still possible in the presence of magnetic fields but either requires strong initial perturbations or must occur during the second collapse phase, after the dissociation of $\mathrm{H}_{2}$ (Machida et al. 2008). The systems formed in the latter case are initially very-low-mass $\left(\sim 0.01 M_{\odot}\right)$, close $(\sim 1 \mathrm{AU})$ binaries (Bonnell \& Bate 1994), which have to grow substantially by accretion during the Class 0/Class I phase (Bate 2000) to match the properties of observed young binary stars (e.g. Duchêne et al. 2004). Therefore, both from an observational and a theoretical point of view, it is unclear whether the collapse of a prestellar core typically produces one, two, or more stars.

The multiplicity of solar-type pre-main-sequence stars and Class II/Class I young stellar objects (YSOs) is now well documented and has been investigated at a range of wavelengths (e.g., Ghez et al. 1993; Simon et al. 1995; Patience et al. 2002; Duchêne et al. 2004, 2007; Connelley et al. 2008). In particular, Patience et al. (2002) and Köhler et al. (2008) showed that the binary frequency of (Class II) $\mathrm{T}$ Tauri stars ranges from $\sim 40 \%$ to $\sim 60 \%$ with a peak in the separation distribution around $60_{-20}^{+40}$ AU. Duchêne et al. (2007) showed that $\sim 32 \%$ of Class I YSOs have companions in the $\sim 50-1000$ AU separation range. The observed Class I/II binary systems have typical mass ratios $q=M_{\text {second }} / M_{\text {main }} \sim 0.2-1$ (e.g. Woitas et al. 2001). Unfortunately, observations of Class II/Class I YSOs barely constrain the pristine properties of multiple systems since dynamical evolution can drastically alter these properties in less than $\sim 10^{5}$ yr (cf. Reipurth \& Clarke 2001). Although still fairly uncertain and a matter of debate, the lifetime of the Class 0 phase is estimated to be $\sim 3 \times 10^{4}-10^{5} \mathrm{yr}$ (André et al. 2000; Evans et al. 2009), compared to $\sim 2-5 \times 10^{5} \mathrm{yr}$ for the Class I phase (Greene et al. 1994; Evans et al. 2009). Moreover, regardless of their precise age and lifetime, Class 0 objects are envelopedominated protostars $\left(M_{\mathrm{env}} \gg M_{\star}-\right.$ André et al. 1993, 2000), while Class I objects tend to have only residual protostellar envelopes $\left(M_{\mathrm{env}}<M_{\star}-\right.$ André \& Montmerle 1994; Motte \& André 2001). Therefore, Class 0 protostars are much more likely than Class I objects to retain detailed information about the collapse initial conditions and the physics of the binary fragmentation process. Furthermore, the circumstellar mass reservoir left around Class I sources is generally not sufficient to form companions more massive than substellar objects at the Class I stage. Probing the multiplicity on scales $<1000 \mathrm{AU}$ as soon as the Class 0 stage is thus one of the keys to understanding the bulk of multiple star formation.

Only subarcsecond $\mathrm{mm} / \mathrm{submm}$ interferometry can probe the inner structure of Class 0 objects, so that little is known about their multiplicity on scales $<150$ AU. Several interferometric studies discovered a number of wide ( $\gtrsim 1000 \mathrm{AU})$ multiple Class 0 systems (Looney et al. 2000; Bourke 2001; Chen et al. 2008; Girart et al. 2009), but these studies were limited by sensitivity to small samples of relatively luminous objects. The SMA has recently been used to study several Class 0 sources at $1.3 \mathrm{~mm}$ and $0.8 \mathrm{~mm}$ but only at $\sim 2^{\prime \prime}$ ( $\left.\sim 450 \mathrm{AU}\right)$ resolution (Jørgensen et al. 2007), which does not allow the detection of tight multiple systems. Among the eight Class 0 sources targeted with the SMA, only one source already embedded in a wide separate-envelope system (NGC 1333-IRAS4A / NGC 1333-IRAS4B) was found to exhibit a higher degree of multiplicity on smaller scales: Jørgensen et al. (2007) showed that IRAS 4A splits into two components separated by $\sim 2^{\prime \prime}$ (450 AU) and IRAS 4B splits into two components separated by $\sim 11^{\prime \prime}(2400 \mathrm{AU})$. These two new companions were detected at both $1.3 \mathrm{~mm}$ and $0.8 \mathrm{~mm}$.

To determine the typical outcome of protostellar collapse and constrain binary fragmentation models, high-resolution imaging of very young protostars observed as early as possible after the end of the first collapse phase are crucially needed. In this paper, we present the results of a pilot high-resolution survey of 5 Class 0 objects carried out with the IRAM Plateau de Bure Interferometer (PdBI) equipped with new-generation $1.3 \mathrm{~mm}$ receivers and using the most extended baselines of the interferometer (new A configuration).

\section{Observations and data reduction}

\subsection{Sample selection}

In order to probe the multiplicity at the Class 0 stage, we conducted a pilot survey of 5 sources with the Plateau de Bure Interferometer (PdBI) in February 2008. The sources were first selected based on a distance criterion: they had to be close enough so that the PdBI resolution in the most extended configuration would probe $\lesssim 150 \mathrm{AU}$ scales, i.e. all sources had to be located at $d<250-300$ pc. The second criterion was the source locations in the sky: the sources had to be visible from the PdBI in winter and observable with a synthetized beam of less than $0.6^{\prime \prime}$ (in both directions) with the A array. The selected sample includes the following five sources: IRAM 04191, L1527, L1521F, all located in Taurus at $d \sim 140 \mathrm{pc}$, and L1448-C and NGC 1333IRAS2A both located in the Perseus cloud at $d \sim 250 \mathrm{pc}$ (see Table 1 ). These 5 sources have $M_{\text {env }} \sim 0.5-4 M_{\odot}, L_{\text {bol }} \lesssim 10 L_{\odot}$ and are among the youngest known solar-type Class 0 protostars (André et al. 2000). The main properties of the 5 Class 0 target sources are summarized below.

\subsubsection{L1448-C}

L1448-C is located in the Perseus molecular cloud, and was first detected as a $2 \mathrm{~cm}$ radio continuum source (Curiel et al. 1990), associated with a strong millimeter continuum source (Bachiller et al. 1991). This is a well-known low-mass Class 0 protostar (Barsony et al. 1998) driving a powerful, highly collimated outflow (Bachiller et al. 1990), which has been imaged at high 
Table 1. Class 0 source sample.

\begin{tabular}{lccccccc}
\hline \hline Source & $\alpha(\mathrm{J} 2000)$ & $\delta(\mathrm{J} 2000)$ & SFR $^{1}$ & $\begin{array}{c}\text { Distance }^{2} \\
(\mathrm{pc})\end{array}$ & $\begin{array}{c}M_{\text {env }} \\
\left(M_{\odot}\right)\end{array}$ & $\begin{array}{c}L_{\text {bol }} \\
\left(L_{\odot}\right)\end{array}$ & Ref. $^{3}$ \\
\hline L1448-C & $03: 25: 38.87$ & $+30: 44: 05.4$ & Perseus & 250 & 1.6 & 5 & $(\mathrm{a})$ \\
NGC 1333-IRS2A & $03: 28: 55.58$ & $+31: 14: 37.1$ & Perseus & 250 & 1.7 & 10 & $(\mathrm{~b})$ \\
IRAM 04191 & $04: 21: 56.91$ & $+15: 29: 46.1$ & Taurus & 140 & $0.5-1.5$ & 0.1 & $(\mathrm{c})$ \\
L1527 & $04: 39: 53.90$ & $+26: 03: 10.0$ & Taurus & 140 & $0.8-1.7$ & 1.6 & $(\mathrm{a}),(\mathrm{d})$ \\
L1521-F & $04: 28: 38.99$ & $+26: 51: 35.6$ & Taurus & 140 & $0.7-4$ & 0.1 & $(\mathrm{e}),(\mathrm{f})$ \\
\hline
\end{tabular}

Notes. ${ }^{(1)}$ Star Forming Region with which the Class 0 object is associated. ${ }^{(2)}$ Recent estimates of the distance to Perseus range from 220 to 350 pc. Throughout this paper, we adopt a distance of $250 \mathrm{pc}$ for the Perseus molecular cloud (Enoch et al. 2006). ${ }^{(3)}$ References for the adopted values of $M_{\text {env }}$ and $L_{\text {bol }}$ : (a) Motte \& André (2001); (b) Jørgensen et al. (2007); (c) André et al. (1999); (d) Ohashi et al. (1997); (e) Bourke et al. (2006); (f) Crapsi et al. (2004).

resolution (synthetized $H P B W$ beam $\sim 2.5^{\prime \prime}$ at $\sim 90 \mathrm{GHz}$ ) in $\mathrm{CO}$ and $\mathrm{SiO}$ with PdBI (Guilloteau et al. 1992; Bachiller et al. 1995). The molecular jet has been extensively studied since its discovery, and is known to exhibit very high-velocity features ( $\pm 70 \mathrm{~km} \mathrm{~s}^{-1}-$ Bachiller et al. 1990, 1995).

\subsubsection{NGC 1333-IRAS2A}

NGC 1333-IRAS2A was first identified in $450 \mu \mathrm{m}$ and $850 \mu \mathrm{m}$ continuum observations (Sandell et al. 1994; Sandell \& Knee 2001), and is also detected at cm-wavelengths (Rodríguez et al. 1999; Reipurth et al. 2002) and as a compact 3 mm continuum source (Jørgensen et al. 2004). CO maps of the IRAS2 region show two outflows, directed north-south and east-west (Liseau et al. 1988; Knee \& Sandell 2000), both originating near IRAS2A. Therefore, it has been argued that IRAS2A may be an unresolved protobinary. However, neither the $2.7 \mathrm{~mm}$ BIMA observations of Looney et al. (2000) nor the $1.3 \mathrm{~mm}$ SMA observations of Jørgensen et al. (2007) detect a companion to the source, despite a $\sim 3 \mathrm{mJy} /$ beam sensitivity in both cases and beam sizes of $0.6^{\prime \prime}$ and $2.2^{\prime \prime}$, respectively.

\subsubsection{IRAM 04191}

The very low luminosity Class 0 object, IRAM 04191+1522 (hereafter IRAM 04191), is located in the southern part of the Taurus molecular cloud and was originally discovered in the millimeter dust continuum (André et al. 1999). Follow-up observations revealed the presence of a $\mathrm{CO}$ bipolar outflow and a weak $3.6 \mathrm{~cm}$ VLA radio continuum source located at its center of symmetry (André et al. 1999), as well as extended infall and rotation motions in a prominent, flattened envelope (Belloche et al. 2002). It is associated with a weak Spitzer source and has an estimated accretion luminosity of only $L_{\text {int }} \sim 0.08 L_{\odot}$ (Dunham et al. 2006).

\subsubsection{L1527}

L1527 IRS (hereafter L1527), located in the Taurus molecular cloud, has been classified as a borderline Class 0/I object. It is observed in a nearly edge-on configuration $\left(\sim 90^{\circ}\right.$ viewing angle $)$ (Ohashi et al. 1997) and features a large, dense circumstellar envelope (Ladd et al. 1991; Chen et al. 1995; Motte \& André 2001). It also exhibits a prominent bipolar outflow whose lobes are oriented along the east-west direction (Parker et al. 1991).

\subsubsection{L1521-F}

L1521-F, located in the Taurus molecular cloud, was originally classified as an evolved starless core (Codella et al. 1997; Onishi et al. 1999; Crapsi et al. 2004). The high central density and infall asymmetry seen in the $\mathrm{HCO}^{+}(3-2)$ line indicate an object in the earliest stages of gravitational collapse (Onishi et al. 1999). ${ }^{12} \mathrm{CO}(2-1)$ observations show no clear evidence of bipolar outflow emission. Recent Spitzer observations of L1521-F detected a low luminosity protostar at mid-infrared wavelengths $(>5 \mu \mathrm{m})$ and in the MIPS $160 \mu \mathrm{m}$ data, which led to a reclassification of this object as a very low luminosity Class 0 object (e.g. Bourke et al. 2006; Terebey et al. 2009). Only scattered light is detected at near-infrared wavelengths with IRAC, in the form of a bipolar nebula oriented east-west which is probably tracing an outflow cavity.

\subsection{IRAM Plateau de Bure observations}

Observations of the five sources were carried out at $1.3 \mathrm{~mm}$ with the IRAM Plateau de Bure Interferometer (PdBI), equipped with new-generation receivers in February 2008 (PdBI project R068). Broad band continuum emission and ${ }^{12} \mathrm{CO}(2-1)$ emission were observed simultaneously, with the PdBI in its most extended configuration (A array with 6 antennas, providing 15 instantaneous baselines ranging from $24 \mathrm{~m}$ to $760 \mathrm{~m}$ ). The proximity of the Taurus and Perseus clouds in the sky allowed us to use the same gain calibrators for the two regions, and therefore time-share two tracks of $\sim 10 \mathrm{~h}$ on the five sources. Each track was divided unequally, depending on the expected fluxes of the sources. In particular, a factor of three more time was spent integrating on IRAM 04191 and L1521-F than on L1448-C. Several nearby phase calibrators (mainly $0415+379$ and $0528+134$ ) were observed to determine the time-dependent complex antenna gains. The correlator bandpass was calibrated on the strong quasars 3C 273 and 3C 454.3, while the absolute flux density scale was derived from MWC349 and 3C 84. The absolute flux calibration uncertainty is estimated to be $\sim 15 \%$. During the observations, one spectral unit of the correlator was tuned to the ${ }^{12} \mathrm{CO}(2-1)$ line at $230.538 \mathrm{GHz}$. The total bandwidth of this ${ }^{12} \mathrm{CO}$ spectral unit was $160 \mathrm{MHz}$, with individual channel spacings of $625 \mathrm{kHz}$ (corresponding to a velocity resolution of $1.62 \mathrm{~km} \mathrm{~s}^{-1}$ ). The remaining six windows of the correlator were combined to observe the continuum emission with a total bandwidth of $1.92 \mathrm{GHz}$ between $229.5 \mathrm{GHz}$ and $231.5 \mathrm{GHz}$. The average system temperature of the $1 \mathrm{~mm}$ receivers was $\sim 250 \mathrm{~K}$. The typical angular resolution was $0.5^{\prime \prime} \times 0.3^{\prime \prime}(H P B W)$ at the 
Table 2. Rms noise levels and naturally-weighted beam sizes of the final maps.

\begin{tabular}{|c|c|c|c|c|c|c|c|c|}
\hline \multirow[t]{2}{*}{ Source } & \multicolumn{2}{|c|}{$230 \mathrm{GHz}$} & \multicolumn{2}{|c|}{$230 \mathrm{GHz}$ combined ${ }^{1}$} & \multicolumn{2}{|c|}{${ }^{12} \mathrm{CO}(2-1)$} & \multicolumn{2}{|c|}{$107 \mathrm{GHz}$} \\
\hline & $H P B W$ & $\begin{array}{c}\mathrm{rms} \\
(\mathrm{mJy} / \text { beam })\end{array}$ & $H P B W$ & $\begin{array}{c}\mathrm{rms} \\
(\mathrm{mJy} / \text { beam })\end{array}$ & $H P B W$ & $\begin{array}{c}\mathrm{rms} \\
(\mathrm{mJy} / \text { beam })\end{array}$ & $H P B W$ & $\begin{array}{c}\mathrm{rms} \\
(\mathrm{mJy} / \text { beam })\end{array}$ \\
\hline L1448-C & $0.48^{\prime \prime} \times 0.27^{\prime \prime}$ & 0.9 & $1.68^{\prime \prime} \times 1.39^{\prime \prime}$ & 2.8 & $0.48^{\prime \prime} \times 0.27^{\prime \prime}$ & 10 & $4.08^{\prime \prime} \times 3.27^{\prime \prime}$ & 10 \\
\hline NGC 1333-IRS2A & $0.49^{\prime \prime} \times 0.32^{\prime \prime}$ & 1.16 & - & - & $0.49^{\prime \prime} \times 0.32^{\prime \prime}$ & 20 & - & - \\
\hline IRAM 04191 & $0.56^{\prime \prime} \times 0.31^{\prime \prime}$ & 0.37 & $1.37^{\prime \prime} \times 0.82^{\prime \prime}$ & 0.31 & $0.56^{\prime \prime} \times 0.31^{\prime \prime}$ & 10 & - & - \\
\hline L1527 & $0.48^{\prime \prime} \times 0.28^{\prime \prime}$ & 1.2 & $0.88^{\prime \prime} \times 0.79^{\prime \prime}$ & 2.0 & $0.48^{\prime \prime} \times 0.28^{\prime \prime}$ & 20 & - & - \\
\hline L1521-F & $0.49^{\prime \prime} \times 0.27^{\prime \prime}$ & 0.12 & - & - & $0.48^{\prime \prime} \times 0.27^{\prime \prime}$ & 8 & - & - \\
\hline
\end{tabular}

Notes. ${ }^{(1)}$ L1448-C and L1527: combination of $230 \mathrm{GHz}$ data from project R068 with $218 \mathrm{GHz}$ data from project G080. IRAM 04191: combination of $230 \mathrm{GHz}$ data from project R068 with $227 \mathrm{GHz}$ data from Belloche et al. (2002).

declinations of the targets, while the full width at half maximum $(F W H M)$ of the PdBI primary beam is $\sim 22^{\prime \prime}$ at $230 \mathrm{GHz}$.

In this study, we also make use of the $1.4 \mathrm{~mm}$ PdBI observations of both L1448-C and L1527, and the $2.8 \mathrm{~mm}$ observations of L1448-C, carried out in the B, C, D configurations between November 1996 and September 1998 (unpublished PdBI project G080 by Motte et al.). The typical resolution of these early PdBI observations was $\sim 4^{\prime \prime}$ at $2.8 \mathrm{~mm}(107 \mathrm{GHz})$ and $\sim 2^{\prime \prime}$ at $1.4 \mathrm{~mm}$ (219 GHz).

In addition, the $227 \mathrm{GHz}$ observations obtained by Belloche et al. (2002) toward IRAM 04191 in the B, C, D configurations of PdBI, which had an $H P B W$ angular resolution $\sim 2^{\prime \prime}$, were also used in combination with our A-configuration observations at $230 \mathrm{GHz}$.

\subsection{Interferometric data reduction}

All the data were calibrated, mapped, and analyzed with the GILDAS $^{1}$ software package. Each map was deconvolved down to the theoretical rms noise level using the MAPPING CLEAN method (Clark 1980). Natural weighting was applied to the measured visibilities, producing synthetized half power beam width $(H P B W)$ resolutions $\sim 0.5^{\prime \prime} \times 0.3^{\prime \prime}$, as given in Table 2 . Note that the $3 \mathrm{~mm}$ continuum maps of L1448-C and L1527 have significantly larger synthetized beams $\left(H P B W \sim 4^{\prime \prime} \times 3.5^{\prime \prime}\right)$ than the $1.3 \mathrm{~mm}$ maps, as no $3 \mathrm{~mm}$ data were obtained with the A-configuration. The restored continuum maps have rms values of 0.12-2.8 mJy/beam (see Table 2), depending on the integration time, array configuration and receivers used during the observations.

Likewise, ${ }^{12} \mathrm{CO}(2-1)$ data cubes were produced with natural uv-weighting, resulting in effective angular resolutions and rms noise values reported in the fourth column block of Table 2.

The 1.3 mm continuum visibilities obtained toward L1448-C and L1527 in the various PdBI configurations were merged together in order to produce high spatial dynamic range maps. Since the A-configuration data were obtained at a central frequency of $230.5 \mathrm{GHz}$, while the BCD-configuration data were obtained at a central frequency of $219 \mathrm{GHz}$, we had to scale the BCD-configuration data to $230.5 \mathrm{GHz}$ assuming a spectral index $\alpha=3$, corresponding to a dust emissivity index $\beta \sim \alpha-2 \sim 1$, for the emission of the inner protostellar environment (inner envelope + disk) traced by our maps. Therefore, the $219 \mathrm{GHz}$ visibilities were scaled by $(230 / 219)^{3} \sim 1.16$ before merging the R068 and G080 datasets at $230.5 \mathrm{GHz}$. The respective weights of each dataset were adjusted so as to produce the highest possible

\footnotetext{
${ }^{1}$ Grenoble Image and Line Data Analysis System, software provided and actively developed by IRAM

http://wWW . iram. fr/IRAMFR/GILDAS.
}

resolution image, while keeping a low rms noise. The synthetized beam sizes and rms sensitivities of the combined data are given in the third column block of Table 2. The resulting maps are shown in Figs. 4 and 7 below, respectively.

Following the same method, the $227 \mathrm{GHz}$ PdBI visibilities taken by Belloche et al. (2002) toward IRAM 04191 and the $230 \mathrm{GHz}$ visibilities from our present A-configuration PdBI observations were also merged, in order to produce a high spatial dynamic range map of IRAM 04191 (see Table 2) at $1.3 \mathrm{~mm}$. The resulting map is shown in Fig. 7a.

\section{Results of the A-configuration PdBI observations}

\subsection{High-resolution $1.3 \mathrm{~mm}$ continuum maps}

The $1.3 \mathrm{~mm}$ dust continuum maps we obtained with the A-array of PdBI toward the 5 sources are shown in Figs. 1 and 2. The effective spatial resolution of these maps is better than $70 \mathrm{AU}$ and $125 \mathrm{AU}(H P B W)$ for the Taurus and Perseus sources, respectively. All five primary Class 0 targets are detected in these highresolution maps, with signal-to-noise ratios ranging from 5.4 (IRAM 04191) to 65 (L1448-C) (see Table 3 for flux densities and source sizes).

The three Class 0 sources of Taurus targeted in the present study are found to be single in our maps: the main protostellar object is the only source detected above the $5 \sigma$ level in each of the maps shown in Fig. 1. A tentative $5 \sigma(\sim 6.6 \mathrm{mJy} / \mathrm{beam})$ secondary component is detected in the L1527 map (3.6" west of the main source), but it is located at the edge of a dirty lobe, which casts doubt on the detection. Moreover, this tentative component is not detected in the combined map shown in Fig. 7 (while such a source should have been detected, given the rms noise level of the combined map). Therefore, we will not mention this source anymore in the following.

On the other hand, both Perseus maps (Figs. 2a and 2b) show evidence of secondary $1.3 \mathrm{~mm}$ continuum components detected above the $5 \sigma$ level. The map of L1448-C reveals a secondary $1.3 \mathrm{~mm}$ continuum source, located $\sim 2.4^{\prime \prime}$ south-east from the primary L1448-C source (Table 3 ). It is the only additional component detected above the $3 \sigma$ level in the whole $22^{\prime \prime}$ map (see Figs. 2 and 3). The map of NGC 1333-IRAS2A (see Fig. 2) also shows a secondary $1.3 \mathrm{~mm}$ continuum source (see position in Table 3) detected above the $5 \sigma$ level. The nature of these secondary sources is discussed further in Sect. 4 below.

\section{2. ${ }^{12} \mathrm{CO}(2-1)$ data}

Compact ${ }^{12} \mathrm{CO}(2-1)$ emission is detected toward all of the targets, except L1521-F. Moreover, significant high-velocity 
(a)

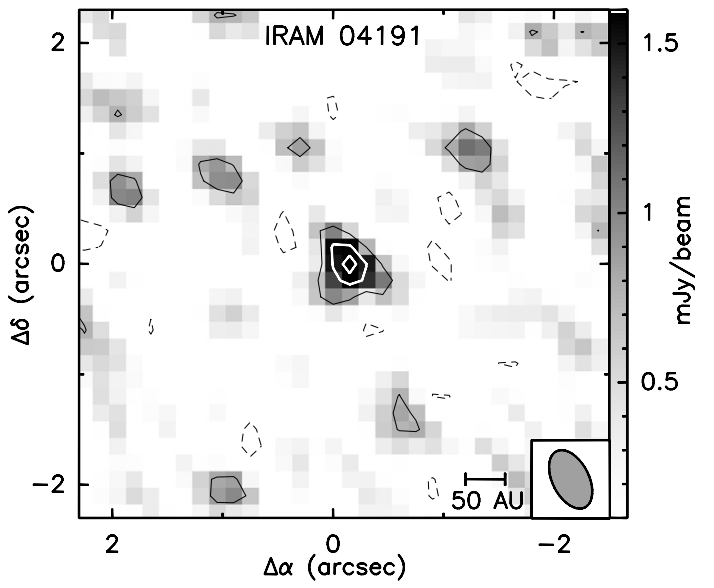

(b)

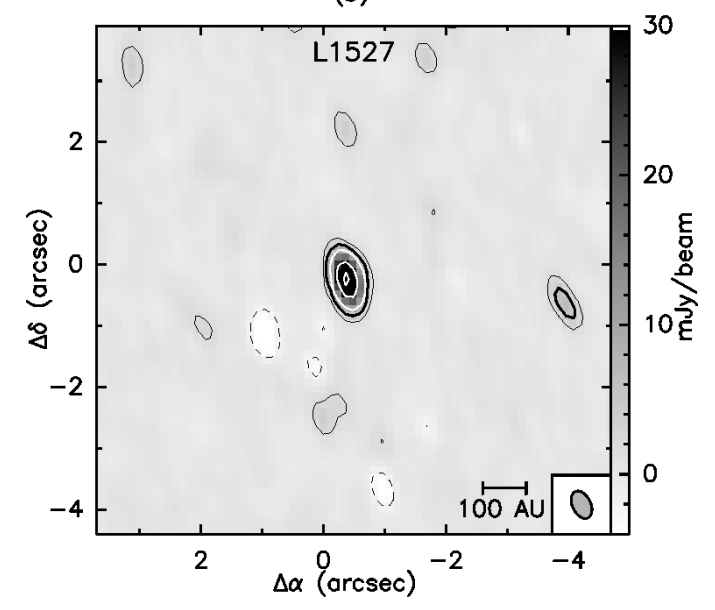

(c)

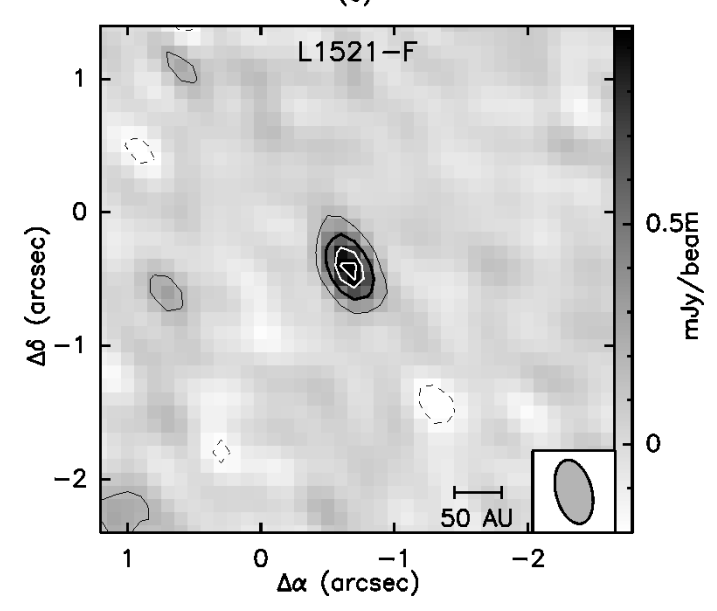

Fig. 1. High resolution $1.3 \mathrm{~mm}$ continuum maps of the Taurus sources. In all panels, the filled ellipse in the bottom right corner shows the synthetized $H P B W$ beam. a) IRAM 04191 . The synthetized $H P B W$ is $0.57^{\prime \prime} \times 0.33^{\prime \prime}$, and the rms noise is $\sigma \sim 0.37 \mathrm{mJy} / \mathrm{beam}$. The contour levels are $-2 \sigma$ (dashed), $2 \sigma, 4 \sigma$ and $5 \sigma$. b) L1527. The $H P B W$ is $0.48^{\prime \prime} \times 0.28^{\prime \prime}$, and $\sigma \sim 1.2 \mathrm{mJy} / \mathrm{beam}$. The contour levels are $-2 \sigma$ (dashed), $2 \sigma$ and $5 \sigma$ (bold), and $10 \sigma, 30 \sigma, 50 \sigma$ in white. c) L1521-F. The HPBW is $0.49^{\prime \prime} \times 0.27^{\prime \prime}$, and $\sigma \sim 0.1 \mathrm{mJy} / \mathrm{beam}$. The contour levels are $-2 \sigma$ (dashed), $2 \sigma, 5 \sigma$ (bold), and $8 \sigma, 10 \sigma$ in white.

(a)

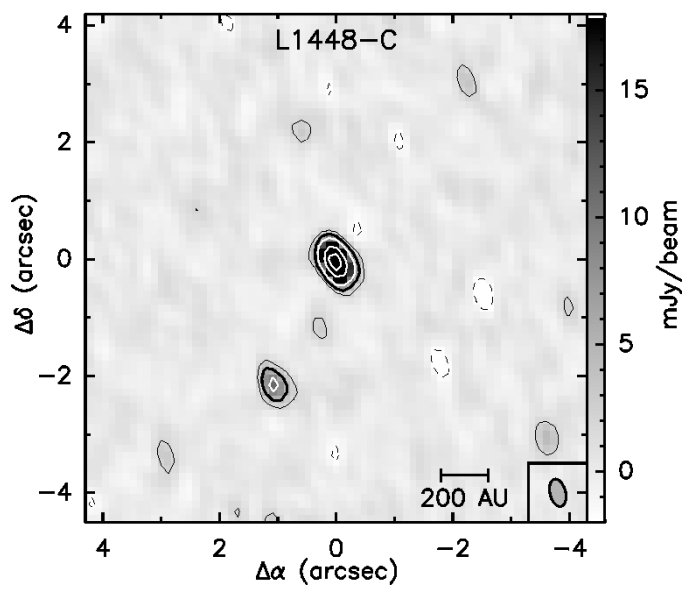

(b)

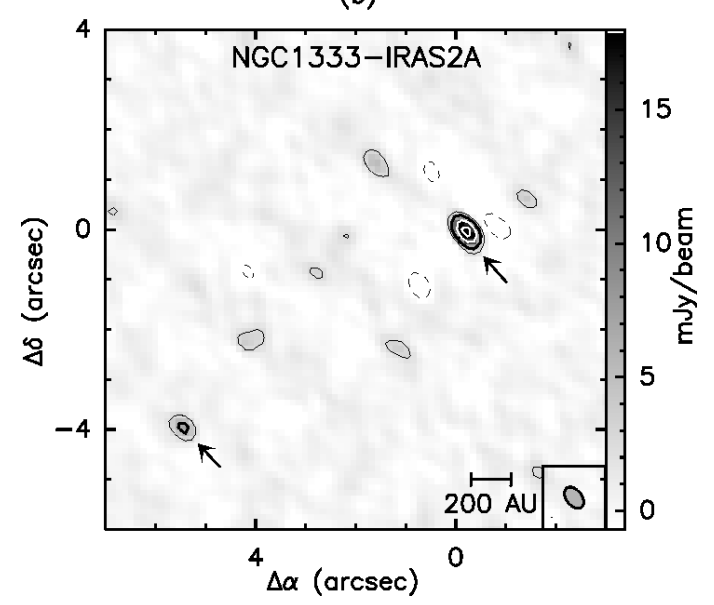

Fig. 2. High resolution $1.3 \mathrm{~mm}$ continuum maps of the Perseus sources. In both panels, the filled ellipse in the bottom right corner indicates the synthesized $H P B W$ beam. a) L1448-C. The $H P B W$ is $0.48^{\prime \prime} \times 0.27^{\prime \prime}$, and the rms noise is $\sigma \sim 0.93 \mathrm{mJy} /$ beam. The contour levels are $-2 \sigma($ dashed), $2 \sigma$, and $5 \sigma$ (bold), $10 \sigma$ to $50 \sigma$ by $20 \sigma$ in white. b) NGC 1333 -IRAS2A. The $H P B W$ beam is $0.57^{\prime \prime} \times 0.33^{\prime \prime}$, and $\sigma \sim 1.16 \mathrm{mJy} / \mathrm{beam}$. The contour levels are $-2 \sigma$ (dashed), $2 \sigma, 5 \sigma$ (bold); and $12 \sigma, 20 \sigma$ in white. The two arrows show the positions of the two sources detected above the $5 \sigma$ level. 
A\&A 512, A40 (2010)

Table 3. Properties of the $1.3 \mathrm{~mm}$ continuum sources detected in the high-resolution PdBI maps.

\begin{tabular}{lcccccc}
\hline \hline Source & \multicolumn{2}{c}{ Position $(1.3 \mathrm{~mm})$} & $\begin{array}{c}\text { Peak flux } \\
(\mathrm{mJy} / \mathrm{beam})\end{array}$ & $\begin{array}{c}F W H M^{1} \\
(\operatorname{arcsec})\end{array}$ & $\begin{array}{c}\text { Flux density } \\
(\mathrm{mJy})\end{array}$ & $\begin{array}{c}\text { Separation }^{3} \\
(\mathrm{AU})\end{array}$ \\
\hline L1448-C & $03: 25: 38.87$ & $30: 44: 05.3$ & $59 \pm 0.9$ & $0.9 \pm 0.05$ & $57 \pm 2$ & - \\
L1448-C / South1 & $03: 25: 38.95$ & $30: 44: 03.2$ & $11 \pm 0.7$ & $0.7 \pm 0.1$ & $11 \pm 2$ & 600 \\
NGC 1333-IRS2A & $03: 28: 55.56$ & $31: 14: 37.1$ & $26 \pm 1.16$ & $0.55 \pm 0.07$ & $18 \pm 2$ & - \\
NGC 1333-IRS2A / SE & $03: 28: 56.00$ & $31: 14: 33.1$ & $7 \pm 1.16$ & $0.23 \pm 0.04$ & $4 \pm 1.5$ & 1900 \\
IRAM 04191 & $04: 21: 56.90$ & $15: 29: 46.1$ & $2 \pm 0.37$ & $0.28 \pm 0.02$ & $0.9 \pm 0.4$ & - \\
L1527 & $04: 39: 53.87$ & $26: 03: 09.8$ & $50 \pm 1.2$ & $0.42 \pm 0.05$ & $65 \pm 3$ & - \\
L1521-F & $04: 28: 38.94$ & $26: 51: 35.2$ & $1 \pm 0.12$ & $0.65 \pm 0.009$ & $0.8 \pm 0.2$ & - \\
\hline
\end{tabular}

Notes. ${ }^{(1)} F W H M$ diameter is computed from a circular Gaussian fit to the visibilities.

(2) Flux integrated above the $3 \sigma$ level.

(3) Projected distance to the primary source.

${ }^{12} \mathrm{CO}(2-1)$ emission is detected only toward L1448-C. This is due to the fact that the A configuration of PdBI filters out most of the extended ${ }^{12} \mathrm{CO}(2-1)$ emission from both protostellar outflows and the parent molecular clouds.

In the ${ }^{12} \mathrm{CO}(2-1)$ map of L1448-C, a total of seven compact high-velocity CO "bullets" are detected along the bipolar jet axis, in both the redshifted and blueshifted lobes (see Fig. 3). Two blueshifted bullets are detected above the $3 \sigma$ level $\left(30 \mathrm{~K} \mathrm{~km} \mathrm{~s}^{-1}\right)$ at LSR velocities ranging from $-60 \mathrm{~km} \mathrm{~s}^{-1}$ to $-40 \mathrm{~km} \mathrm{~s}^{-1}$, and are located 1.44" (360 AU) and 2.69" (670 AU) away from the primary source driving the jet, respectively. Five redshifted bullets are detected along the redshifted jet axis, south-west of the driving source. These five features have LSR velocities ranging from $50 \mathrm{~km} \mathrm{~s}^{-1}$ to $80 \mathrm{~km} \mathrm{~s}^{-1}$, and are located at distances from the driving source ranging from $0.9^{\prime \prime}$ (125 AU) to 6.7" (940 AU). The seven high-velocity features detected in the L1448-C map are all remarkably well aligned with the axis of the molecular jet already mapped at lower resolution by Bachiller et al. (1995) with PdBI, and more recently by Jorgensen et al. (2007) with SMA. Therefore, we conclude that these high-velocity bullets trace the inner part of the jet driven by the protostellar source L1448-C.

\section{Nature of the secondary components detected in the millimeter continuum maps}

\subsection{Sources detected in the vicinity of L1448-C}

\subsubsection{Secondary $1.3 \mathrm{~mm}$ continuum source}

In the high resolution $1.3 \mathrm{~mm}$ continuum map of L1448-C shown in Fig. 2a, a secondary source is detected $\sim 2.4^{\prime \prime}$ south-east of the main source. In the combined $1.3 \mathrm{~mm}$ continuum map, this secondary component is no longer resolved from the primary source (see Fig. 4), because its peak flux (11.3 mJy/beam) is only between the $3 \sigma$ and $5 \sigma$ levels, and it is confused with extended $1.3 \mathrm{~mm}$ emission south-east of L1448-C, which likely arises from a cavity in the red-shifted outflow lobe. This secondary source is not detected either in the $3 \mathrm{~mm}$ map shown in Fig. $4 \mathrm{~b}$ due to insufficient angular resolution. But it lies close to the L1448-C jet axis and is immediately adjacent to the second redshifted high-velocity bullet detected in our ${ }^{12} \mathrm{CO}(2-1)$ observations (only $\sim 0.5^{\prime \prime}$ separation - see Fig. 3 ). Furthermore, this secondary $1.3 \mathrm{~mm}$ continuum source coincides with the position of the first $\mathrm{SiO}(2-1)$ peak (clump RI) detected by Guilloteau et al. (1992) in the red-shifted outflow lobe. This traces the presence of an outflow-induced shock at this position, with a high LSR velocity offset of $+50 \mathrm{~km} \mathrm{~s}^{-1}$ (see Fig. 5b). Therefore, we conclude that the secondary $1.3 \mathrm{~mm}$ source detected south-east of L1448-C is not a genuine protostellar companion but rather an outflow feature directly associated with the high-velocity jet from the primary Class 0 object.

\subsubsection{Secondary $3 \mathrm{~mm}$ source and adjacent Spitzer source}

Figure 4 b shows a $3 \mathrm{~mm}(107 \mathrm{GHz})$ continuum map of L1448-C based on BCD-array data taken with PdBI in 1997 (see Sect. 2.2 for details). It reveals the presence of a secondary $3 \mathrm{~mm}$ continuum source, clearly detected above the $5 \sigma$ level at position $\left(03 \mathrm{~h} 25 \mathrm{~m} 39.10 \mathrm{~s},+30^{\circ} 43^{\prime} 57.8^{\prime \prime}\right)$, i.e. $\sim 8.4^{\prime \prime}$ south-east of the main L1448-C source, and which does not have any significant $1.3 \mathrm{~mm}$ counterpart in Fig. 4a. A mid-infrared source was recently detected with Spitzer $0.6 \pm 0.2^{\prime \prime}$ north of this secondary $3 \mathrm{~mm}$ source (Jørgensen et al. 2006), and $\sim 7.8^{\prime \prime}$ south of L1448-C. The small angular separation between the PdBI $3 \mathrm{~mm}$ source and the Spitzer mid-infrared source suggests that they are physically related. Neither of our $1.3 \mathrm{~mm}$ continuum maps shows counterparts to the Spitzer or the $3 \mathrm{~mm}$ source above the $3 \sigma$ level, corresponding to upper limits to the $1.3 \mathrm{~mm}$ peak flux density of $3 \mathrm{mJy} / 0.48^{\prime \prime} \times 0.27^{\prime \prime}$ beam in the high resolution $1.3 \mathrm{~mm}$ map of L1448-C, and $8.4 \mathrm{mJy} / 1.68^{\prime \prime} \times 1.39^{\prime \prime}$ beam in the combined $1.3 \mathrm{~mm}$ map. The non detection of the $3 \mathrm{~mm}$ source at $1.3 \mathrm{~mm}$ implies that the spectral index of the emission is $\alpha_{3 \mathrm{~mm}}^{1.3 \mathrm{~mm}}<2.0$ (after scaling the $1.3 \mathrm{~mm}$ and $3 \mathrm{~mm}$ fluxes to matching beams), excluding the possibility that the whole millimeter emission is due to dust continuum emission from an embedded protostellar object (in this case $\alpha=\beta+2$, where $\beta$ is the dust emissivity index, and $\alpha_{3 \mathrm{~mm}}^{1.3 \mathrm{~mm}} \sim 3-4$ is expected - e.g. Dent et al. 1998).

If the $3 \mathrm{~mm}$ source corresponds to a protostellar object, its non-detection in the $1.3 \mathrm{~mm}$ continuum emission is unlikely to result from interferometric filtering since Class 0 sources have strongly centrally condensed envelopes (André et al. 2000; Motte \& André 2001), and are thus expected to be detected in a $\sim 1.5^{\prime \prime}$ beam. For instance, the $1.3 \mathrm{~mm}$ peak flux density of L1448-C is $\sim 134 \mathrm{mJy} /$ beam in a $\sim 1.5^{\prime \prime}$ beam, so the $3 \mathrm{~mm}$ secondary source would have to be 17 times weaker at $1.3 \mathrm{~mm}$ on $\sim 375 \mathrm{AU}$ scales to be undetected (above $3 \sigma$ level i.e. $8.4 \mathrm{mJy} /$ beam), which is more than twice the flux ratio $\sim 8$ measured between the two sources computed at $3 \mathrm{~mm}$. To further test whether the Spitzer and $3 \mathrm{~mm}$ continuum emission may originate from a protostellar object, we compared the observations with the publicly available grid of model YSO spectral energy distributions (SEDs) published by Robitaille et al. (2007). We tried to reproduce both the mid-infrared fluxes derived by Jørgensen et al. (2006) for the southern Spitzer source and the 


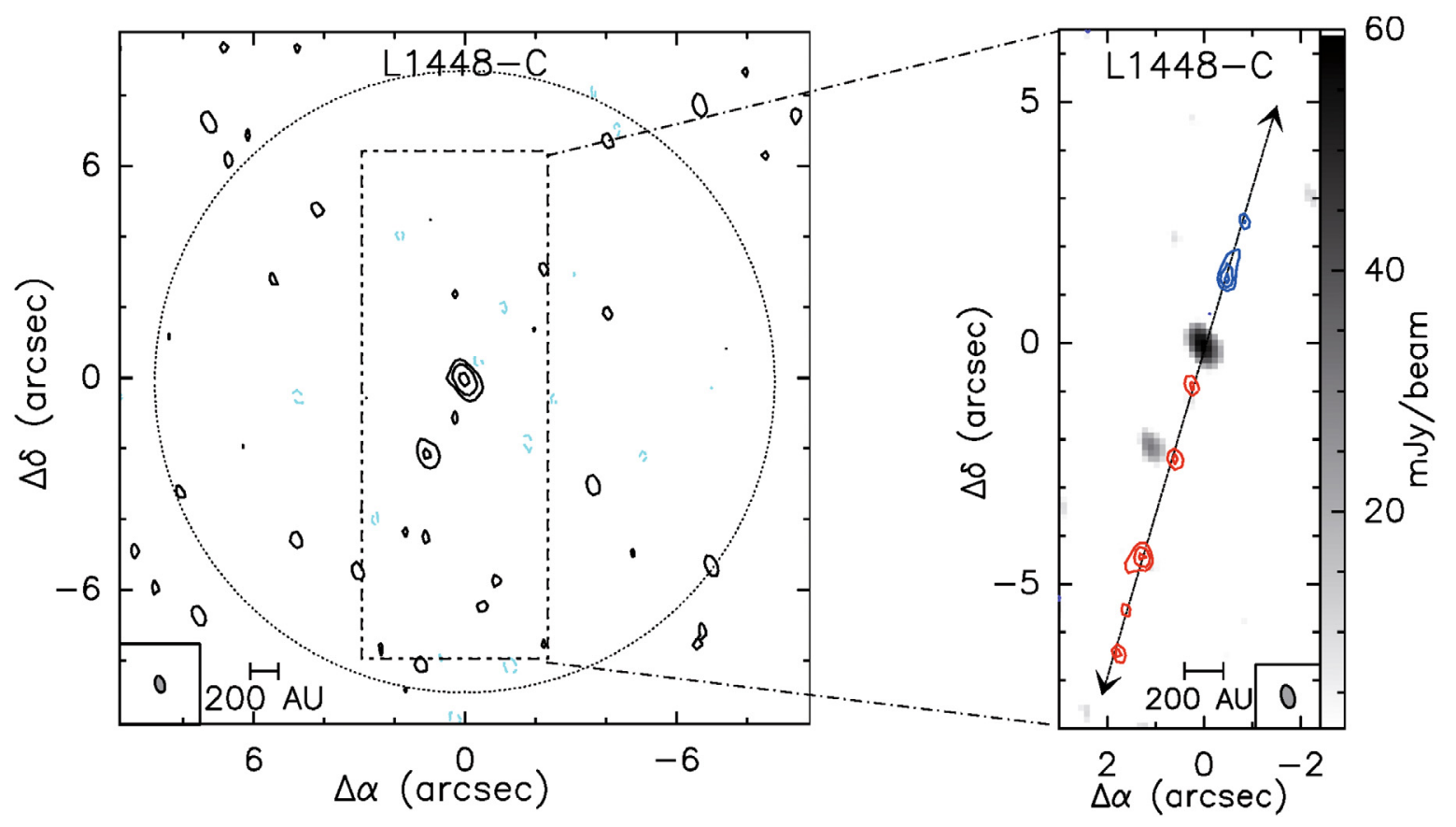

Fig. 3. The left panel shows the high-resolution $1.3 \mathrm{~mm}$ map of L1448-C (same as Fig. 2 but showing a wider area covering most of the primary beam). The large dotted circle represents the cleaned area of the map. Note that the large-scale map has not been corrected for primary beam attenuation at large distances from the centre $\left(F W H M=22^{\prime \prime}\right.$ ). The contour levels are $-2 \sigma$ (light blue dashed), $2 \sigma, 8 \sigma$ and $40 \sigma$. The right panel shows a blow-up of the central part of the high-resolution $1.3 \mathrm{~mm}$ map of L1448-C in greyscale, with superimposed contours of the high-velocity ${ }^{12} \mathrm{CO}(2-1)$ emission integrated from -60 to $-40 \mathrm{~km} \mathrm{~s}^{-1}$ (blue contours) and from +50 to $+80 \mathrm{~km} \mathrm{~s}^{-1}$ (red contours). The first ${ }^{12} \mathrm{CO}(2-1)$ contour corresponds to the $3 \sigma$ level (30 mJy/beam); the next contour levels are $5 \sigma$ and $10 \sigma$. The double arrow marks the direction of the high-velocity jet detected in ${ }^{12} \mathrm{CO}(2-1)$. The filled ellipse at the bottom of the panels indicates the synthesized $H P B W$ resolution.

PdBI $3 \mathrm{~mm}$ flux, while keeping the $1.3 \mathrm{~mm}$ flux density lower than the $5 \sigma$ detection level ( $\sim 5 \mathrm{mJy}$ in a $0.37^{\prime \prime}$ radius aperture) achieved with PdBI. None of the models explored in this way can reproduce the data points properly: the ten best models have total $\chi^{2}$ values $\sim 100-200$ for five data points (compared to total $\chi^{2} \sim 20-40$ with data at the same five wavelengths for L1448C), and show $1.3 \mathrm{~mm}$ fluxes which should have been detected above the $3 \sigma$ level in our PdBI observations. The mid-infrared emission detected with Spitzer and the adjacent $3 \mathrm{~mm}$ emission detected with PdBI are therefore unlikely to originate from a protostellar object.

The $3 \mathrm{~mm}$ and Spitzer sources are both located in the walls of an outflow cavity (see Jorgensen et al. 2007). They both coincide with the second $\mathrm{SiO}(2-1)$ emission peak detected toward the red-shifted lobe (clump RII in Guilloteau et al. 1992) which reveals the presence of an outflow-induced shock at this location, at a high LSR velocity offset of $+65 \mathrm{~km} \mathrm{~s}^{-1}$ (see Fig. 5a). Furthermore, these two sources also coincide with a peak in the $\mathrm{NH}_{3}(2,2) / \mathrm{NH}_{3}(1,1)$ ratio, which traces heating due to the interaction between the energetic outflow and the ambient molecular gas (Curiel et al. 1999). We therefore propose that these two adjacent sources, located along the jet axis, are in fact shockgenerated features (cf. Hartigan 2003) associated with the powerful outflow driven by L1448-C. Compact mid-infrared continuum emission along protostellar jets has already been observed: Lefloch et al. (2005) reported the detection of such features along the HH 2 protostellar jet, and argued that the mid-IR emission arises from heating of very small grains formed by evaporation of dust grain mantles in outflow-induced shocks. Furthermore, the lack of a $1.3 \mathrm{~mm}$ counterpart to the $3 \mathrm{~mm}$ source can be explained by the nature of the $3 \mathrm{~mm}$ emission. The non-detection of VLA $2 \mathrm{~cm}$ emission by Curiel et al. (1990) at the position of the $3 \mathrm{~mm}$ emission implies a spectral index $\alpha_{2 \mathrm{~cm}}^{3 \mathrm{~mm}}>1.5$. Combined with $\alpha_{3 \mathrm{~mm}}^{1.3 \mathrm{~mm}}<2.0$, this suggests that the $3 \mathrm{~mm}$ continuum emission is a combination of optically thick free-free emission $\left(1 \lesssim \alpha_{2 \mathrm{~cm}}^{3 \mathrm{~mm}} \leq 2-\right.$ e.g. Ghavamian \& Hartigan 1998) and optically thin dust continuum emission $\left(\alpha_{3 \mathrm{~mm}}^{1.3 \mathrm{~mm}} \sim 2-4\right)$ associated with a shock in the L1448-C outflow. The fact that this outflow-induced shock (traced by the Spitzer and $3 \mathrm{~mm}$ sources) is not detected at $2 \mu \mathrm{m}$ can be ascribed to high visual extinction toward the southern lobe $\left(A_{\mathrm{V}} \sim 32-\right.$ Dionatos et al. 2009).

Based on this multiwavelength analysis, we conclude that the Spitzer mid-infrared source and the PdBI $3 \mathrm{~mm}$ source detected $\sim 8^{\prime \prime}$ south of L1448-C do not correspond to protostellar objects but are both tracing heating and compression resulting from an oblique shock on the outflow cavity wall.

\subsection{Secondary $1.3 \mathrm{~mm}$ continuum source detected near NGC 1333-IRAS2A}

Our high resolution PdBI observations of NGC 1333-IRAS2A allow us to probe the circumstellar environment of this source down to $\sim 90$ AU scales, with better sensitivity than that achieved in previous interferometric observations. While no secondary component is detected above the $2 \sigma$ level within a radius of $1000 \mathrm{AU}\left(\sim 4^{\prime \prime}\right)$ from the primary source, a new secondary $1.3 \mathrm{~mm}$ component is detected 7.7" ( 1900 AU) south-east of NGC 1333-IRS2A, with a peak flux of $\sim 7 \mathrm{mJy} / \mathrm{beam}$ (see Fig. 2b). This secondary $1.3 \mathrm{~mm}$ source is located near the east-west outflow originating from the vicinity of IRAS2A (see Fig. 6), but is not associated with any known high-velocity CO bullet or shock feature. Therefore, the nature of this source is unclear: it may either be a genuine protostellar companion or 

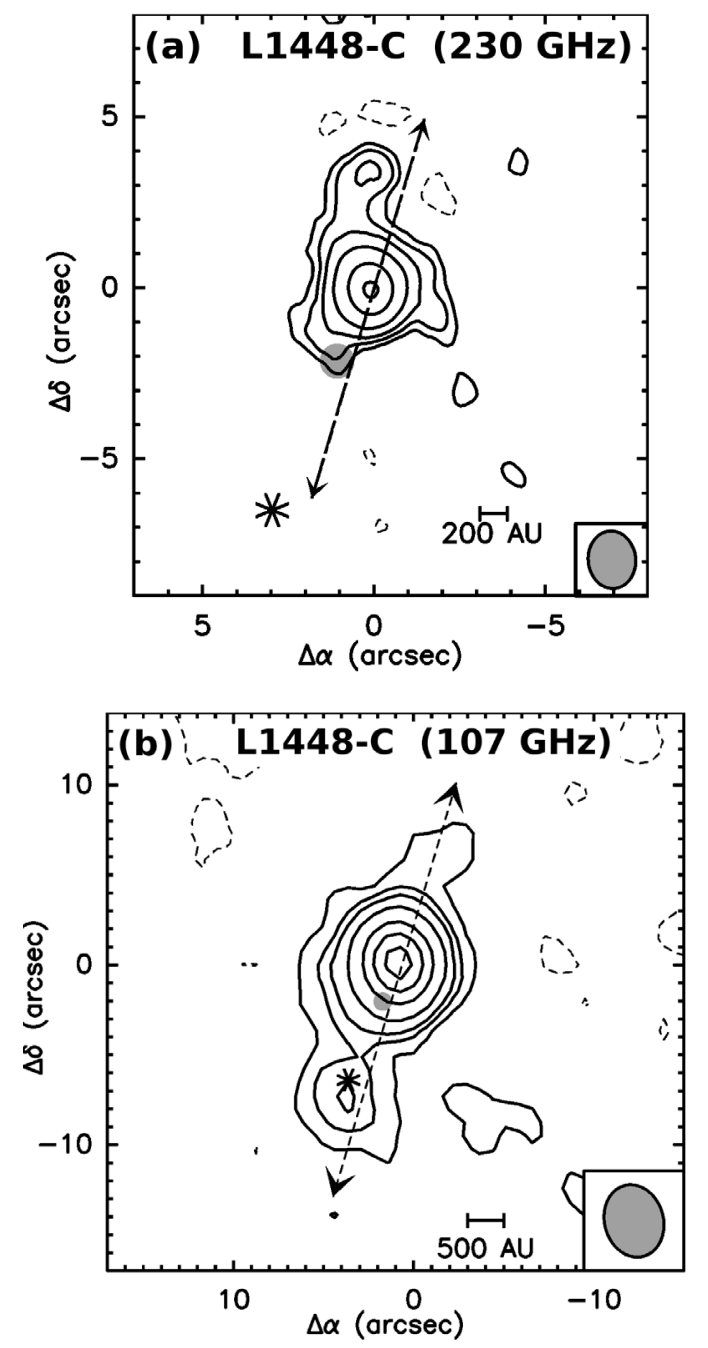

Fig. 4. a) Combined $1.3 \mathrm{~mm}$ dust continuum map of L1448-C. This image was constructed by combining the $230 \mathrm{GHz}$ visibilities obtained with A-configuration of PdBI with the $219 \mathrm{GHz}$ visibilities obtained with the $\mathrm{B}, \mathrm{C}$ and $\mathrm{D}$-configurations of PdBI. The synthetized $H P B W$ is $1.68^{\prime \prime} \times 1.39^{\prime \prime}$, and the rms noise is $\sigma$ is $\sim 2.8 \mathrm{mJy} / \mathrm{beam}$. The dashed contours are levels of $-3 \sigma$. The full contours are levels of $3 \sigma, 5 \sigma, 8 \sigma$ and $15 \sigma$ to $45 \sigma$ by $15 \sigma$. The grey circle indicates the position of the secondary $1.3 \mathrm{~mm}$ continuum source detected in the high resolution map shown in Fig. 2. The arrows indicate the direction of the high-velocity jet observed in ${ }^{12} \mathrm{CO}(2-1)$ (see Sect. 3.2). b) $107 \mathrm{GHz}$ continuum map of L1448-C. The synthetized $H P B W$ is $4.08^{\prime \prime} \times 3.27^{\prime \prime}$, and the rms noise is $\sigma \sim 0.49 \mathrm{mJy} / \mathrm{beam}$. The dashed contour level is $-2 \sigma$. The full contours are levels of $2 \sigma, 5 \sigma, 8 \sigma$ and $15 \sigma$ to $85 \sigma$ by $15 \sigma$. In both panels, the filled ellipses in the lower right corner indicate the synthesized $H P B W$. The star indicates the position of the Spitzer source reported by Jørgensen et al. (2006).

an outflow feature. We name this secondary source NGC 1333IRS2A/SE, as it probably lies within the same envelope as NGC 1333-IRS2A. The weakness of this source explains its nondetection in previous millimeter interferometric studies (Looney et al. 2000; Jørgensen et al. 2007).

\section{Dust continuum emission detected toward the primary Class 0 sources}

A detailed discussion of the small scale properties and detailed morphology of the dust emission detected toward the primary
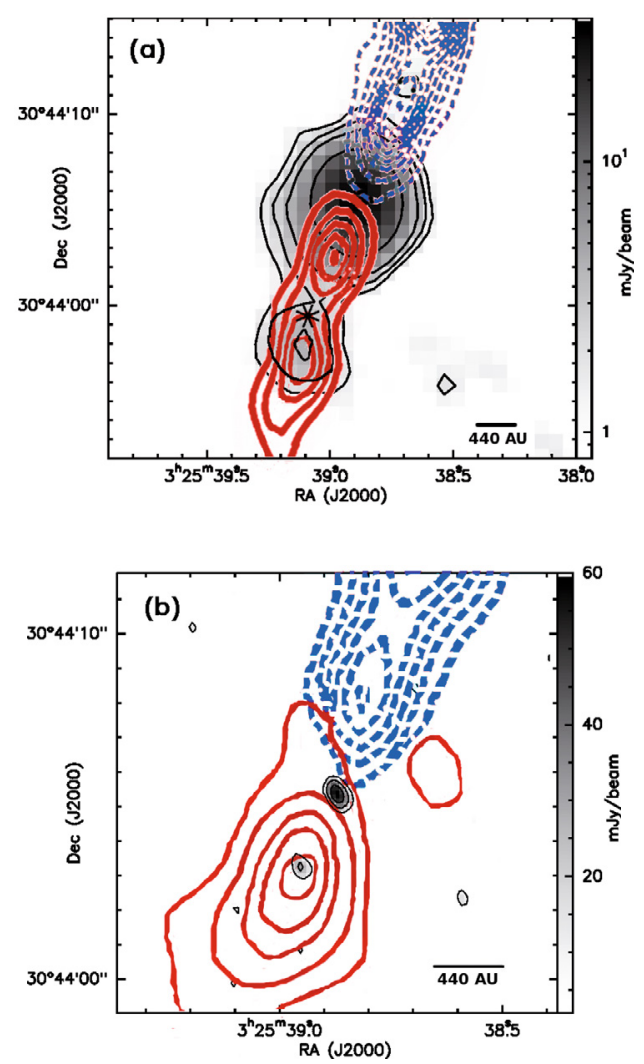

Fig. 5. a) Image and black contours show the $3 \mathrm{~mm}$ continuum map of L1448-C (same as Fig. 4). The red and blue contours are levels of the $\mathrm{SiO}(2-1)$ line intensity at $\pm 65 \mathrm{~km} \mathrm{~s}^{-1}$ from Guilloteau et al. (1992). The star indicates the position of the Spitzer source reported by Jorgensen et al. (2006), for which no counterpart is detected above the $3 \sigma$ level in the $1.3 \mathrm{~mm}$ map (see Sect. 4.1 for a discussion). b) Image and black contours show the high resolution $1.3 \mathrm{~mm}$ map of L1448-C. The red and blue contours are levels of $\mathrm{SiO}(2-1)$ line intensity at $\pm 50 \mathrm{~km} \mathrm{~s}^{-1}$. One can see that both secondary $(1.3 \mathrm{~mm}$ and $3 \mathrm{~mm})$ sources coincide with peaks of $\mathrm{SiO}(2-1)$ emission.

Class 0 sources is beyond the scope of this paper and will be the subject of a forthcoming paper. Here, we provide a simple, qualitative description of the slightly extended $1.3 \mathrm{~mm}$ continuum emission detected with PdBI toward the primary Class 0 sources of our sample.

Our high-resolution $1.3 \mathrm{~mm}$ continuum maps exhibit only little extended emission. This can be explained by the very high resolution achieved in these interferometer maps and the lack of short-spacing data, which filters out most of the extended emission from the envelope material. In the combined $1.3 \mathrm{~mm}$ and $3 \mathrm{~mm}$ maps, however, the use of multiple array configurations allows us to recover some of the extended emission on scales ranging from $\sim 100 \mathrm{AU}$ to $\sim 500 \mathrm{AU}$. Note that, as the older PdBI observations toward L1448-C and L1527 (G080 data taken in the B, C, D configurations) are noisier, the combined $1.3 \mathrm{~mm}$ maps have an improved spatial dynamic range (facilitating, e.g., image reconstruction), but higher rms noise values. The combined $1.3 \mathrm{~mm}$ maps of the two sources IRAM 04191 and L1527 are shown in Figs. 7a and 7b, respectively, while the combined $1.3 \mathrm{~mm}$ map of L1448-C was already shown in Fig. 4a above. Details about the combination procedure can be found in Sect. 2.3 above.

All three combined $1.3 \mathrm{~mm}$ continuum maps show extended emission features, which correlate well with the outflow cavity 


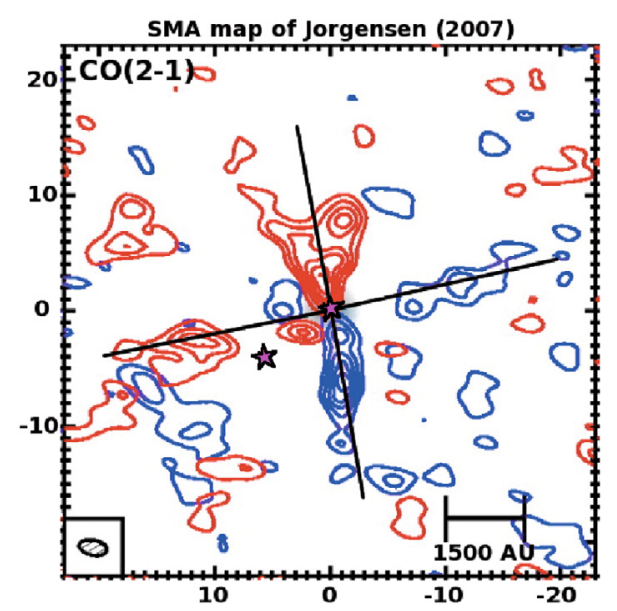

Fig. 6. SMA map obtained in ${ }^{12} \mathrm{CO}(2-1)$ by Jørgensen et al. (2007) toward NGC 1333-IRS2A. The color contours show ${ }^{12} \mathrm{CO}(2-1)$ integrated intensity levels in steps of $3 \sigma$ (see Jørgensen et al. (2007) for details), with the blue contours indicating emission integrated from -6 to $-1 \mathrm{~km} \mathrm{~s}^{-1}$ relative to the systemic velocity, and red contours emission integrated from +1 to $+6 \mathrm{~km} \mathrm{~s}^{-1}$ relative to the systemic velocity. The two lines mark the direction of the protostellar outflows originating near IRAS2A. On large scales, the east-west outflow is more collimated than the $\sim$ north-south bipolar one, and was found to be less energetic than the north-south bipolar outflow (Knee \& Sandell 2000). The two stars mark the position of the $1.3 \mathrm{~mm}$ continuum sources detected in our PdBI observations.
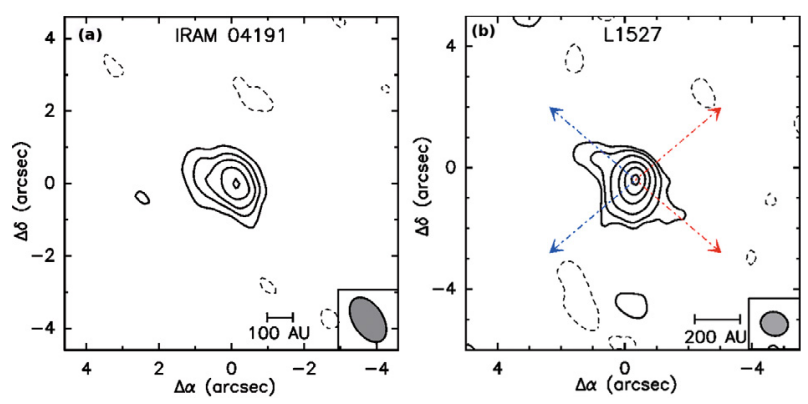

Fig. 7. Combined $1.3 \mathrm{~mm}$ dust continuum maps of the Taurus sources IRAM 04191 and L1527. The filled ellipses in the lower right corner of the panels indicate the synthesized $H P B W$ beam. a) IRAM 04191 . The synthetized beam $(H P B W)$ is $1.37^{\prime \prime} \times 0.82^{\prime \prime}$, and the $1 \sigma$ noise level is $\sim 0.31 \mathrm{mJy} / \mathrm{beam}$. The level of the dashed contours is $-3 \sigma$. The solid contours correspond to levels of $3 \sigma, 5 \sigma, 7 \sigma, 10 \sigma$, and $13 \sigma$. b) L1527. $H P B W$ is $0.88^{\prime \prime} \times 0.79^{\prime \prime}$, and $\sigma$ is $\sim 2.0 \mathrm{mJy} /$ beam. The dashed contour level is $-3 \sigma$. The solid contours are levels of $3 \sigma, 5 \sigma$, and $10 \sigma$ to $40 \sigma$ by $10 \sigma$. The four arrows indicate the cavity walls of the bipolar outflow driven by $\mathrm{L} 1527$, as traced by the emission maps of ${ }^{12} \mathrm{CO}(3-2)$ and $\mathrm{HCO}^{+}(1-0)$ (Hogerheijde et al. 1998).

walls delineated by CO line observations of these Class 0 objects at similar angular resolution (see, e.g., Jørgensen et al. 2007 for a ${ }^{12} \mathrm{CO}(2-1)$ map of L1448-C with an angular resolution comparable to that of our combined $1.3 \mathrm{~mm}$ continuum map). This is particularly clear in the combined $1.3 \mathrm{~mm}$ continuum map of L1527 shown in Fig. 7b, where the extended dust emission around the protostellar source delineates three arms of a cross (see also Motte \& André 2001). The cross-like pattern seen toward L1527 coincides very well with the edges of the outflow cavity traced by $\mathrm{CO}(3-2)$ observations (Hogerheijde et al. 1998; Chandler \& Richer 2000). Furthermore, if we compare our $1.3 \mathrm{~mm}$ continuum map (Fig. $7 \mathrm{~b}$ ) with the $\mathrm{HCO}^{+}(1-0)$ interferometric map of Hogerheijde et al. (1998), we find that the cross-like morphology of the dust continuum emission coincides very closely with the features detected in $\mathrm{HCO}^{+}$. A similar crosslike pattern for the dust continuum emission was also observed by Fuller et al. (1995) toward L1551-IRS5.

This indicates that at least some of the dust continuum emission observed in the immediate vicinity of Class 0 protostars is caused by column density enhancements due to compression in the cavity walls of their outflows.

\section{Discussion: constraints on the formation of multiple systems}

\subsection{Multiplicity rate of Class 0 protostars on $\sim 100 \mathrm{AU}$ scales}

All 5 Class 0 protostars observed in the present pilot PdBI survey are single on scales between $\sim 75$ AU and 1900 AU. The only possible companion found is NGC1333-IRAS2A/SE, which is located $\sim 1900$ AU away from the primary source NGC1333-IRAS2A. We discuss below four possible explanations to the non-detection of close protobinary systems in our sample: (1) small sample statistics; (2) selection effects in our sample; (3) limited mass (and mass ratio) sensitivity; (4) intrinsically small multiplicity fraction for Class 0 protostars on $75-1900$ AU scales.

On the first point, even though we observed a small number of objects, the probability of drawing five single protostars from a binary fraction distribution of $\sim 32 \%$, corresponding to the binary fraction of Class I YSOs in the same separation range (Duchêne et al. 2007), is only $\sim 14 \%$. This suggests that the Class 0 binary fraction may be lower than that of Class I and Class II YSOs, but only with very marginal statistical significance ( $1.5 \sigma$ confidence level) at the present stage. Interestingly, based on an extensive study of 189 Class I sources, Connelley et al. (2008) recently speculated that the opposite trend would be observed, namely that the companion star fraction should be larger at the Class 0 stage.

On the second point, the five sources observed in this study were selected based on a distance criterion mainly, and belong to two different star-forming regions. The sources in our sample have bolometric luminosities ranging from $0.1 L_{\odot}$ to $10 L_{\odot}$, suggesting that they span a relatively wide range of final stellar masses. Moreover, our sample includes two Class 0 objects embedded in clustered environments and belonging to wide systems (L1448-C, NGC 1333-IRAS2A), as well as three relatively isolated objects (IRAM 04191, L1521-F, and L1527). Albeit limited by its small size, our sample thus avoids the most obvious selection biases.

On the third point, our mass sensitivity is directly determined by the rms noise achieved in our high-resolution $1.3 \mathrm{~mm}$ continuum maps. If we assume that the envelope of any putative protostellar companion has similar density and temperature profiles to the envelope of the primary Class 0 object, our observations are sensitive to low circumstellar mass ratios ( $q=M_{\text {second }} / M_{\text {main }} \sim 0.07$ ), except for the faintest two sources, IRAM 04191 and L1521-F, toward which we are only sensitive to $M_{\text {second }} / M_{\text {main }} \sim 0.6-0.9$. Assuming Class 0 systems have a distribution of circumstellar mass ratios similar to the distribution of stellar mass ratios observed toward T-Tauri binary systems (Woitas et al. 2001), we estimate that the sensitivity of our PdBI observations should allow us to detect $\geq 50 \%$ of the Class 0 binary systems with separations wider than $\sim 100 \mathrm{AU}$.

On the fourth and final point, the multiplicity of Class 0 objects on scales $\sim 75-1000$ AU is not well known. While our sample of Class 0 protostars does not show any close multiple 
system (with separations <1900 AU), the early BIMA $2.7 \mathrm{~mm}$ continuum survey by Looney et al. (2000) revealed a higher multiplicity rate in their sample at $2.7 \mathrm{~mm}$. Among the nine Class 0 objects with separate envelopes observed by Looney et al. (2000), three close binary systems were found with separations $<1000$ AU, leading to a binary fraction of $\sim 33 \%$ on scales between $100 \mathrm{AU}$ and $2000 \mathrm{AU}$. We stress, however, that some of the protostellar companions detected by Looney et al. (2000) at $2.7 \mathrm{~mm}$ could in fact be outflow features, like the secondary $3 \mathrm{~mm}$ source detected in our $3 \mathrm{~mm}$ continuum map of L1448-C (Fig. 4b). One striking example is the prototypical Class 0 protostar VLA 1623, for which Looney et al. (2000) detected two $2.7 \mathrm{~mm}$ components separated by $1.11^{\prime \prime}$ (i.e. $\sim 150 \mathrm{AU}$ ), which they interpreted as a proto-binary system. Comparing the highresolution $3.6 \mathrm{~cm}$ VLA image of Bontemps \& André (1997) with the BIMA $2.7 \mathrm{~mm}$ image, it appears that the western $2.7 \mathrm{~mm}$ BIMA component may be associated with an $\mathrm{HH}$-like object named HH-A by Bontemps \& André (1997), belonging to a series of $\mathrm{HH}$-like $\mathrm{cm}$ radio continuum knots almost aligned with the outflow axis. This suggests that the BIMA $2.7 \mathrm{~mm}$ emission observed toward the western component (labeled VLA 1623B in Looney et al. 2000) may be strongly contaminated or even dominated by free-free emission, and may not trace the presence of a bona-fide protostellar companion. In the light of our PdBI findings for L1448-C, careful comparison of the results with the location of the outflows from the primary protostars and detection of both $1 \mathrm{~mm}$ and $3 \mathrm{~mm}$ counterparts (so that a spectral index can be derived in the millimeter range) are needed before the protostellar nature of secondary components detected in millimeter continuum surveys can be firmly established.

Combining the results of the BIMA $2.7 \mathrm{~mm}$ survey of Looney et al. (2000) with our PdBI $1.3 \mathrm{~mm}$ results allows us to enlarge the sample of Class 0 sources for which the multiplicity rate between $\sim 150 \mathrm{AU}$ and $\sim 1000$ AU can be discussed. Based on the above-mentioned arguments, we do not take into account the secondary $3 \mathrm{~mm}$ component detected by Looney et al. (2000) close to VLA 1623, as it is probably an outflow feature similar to the one observed along the outflow axis of L1448-C. The BIMA $2.7 \mathrm{~mm}$ observations probe multiplicity on scales $\gtrsim 100-4000$ AU for Taurus and Ophiuchus sources, and $\gtrsim 150-5000$ AU for Perseus sources. We stress that the enlarged (PdBI + BIMA) sample is not homogeneous because the BIMA and PdBI surveys have differing sensitivities, resolutions, and observing frequencies. Nevertheless, a simple merging of the two samples allows us to draw interesting conclusions. Among the 9 Class 0 objects mapped with BIMA, two of them are binary systems with separations less than 1000 AU: (NGC 1333-IRAS4A1 / NGC 1333-IRAS4A2) and (IRAS 16293-2422A/ IRAS 16293-2422B), while no protobinary system is detected at separations less than 500 AU. Since the merged sample (PdBI+BIMA) has 14 target sources, this leads to estimates of $\sim 14 \%$ for the binary fraction of Class 0 protostars on $\sim 150-1000$ AU scales, and $\$ 7 \%$ for the binary fraction of Class 0 protostars on $\sim 150-550$ AU scales. Assuming that the intrinsic binary fraction of Class 0 objects in the separation range $\sim 150-1000 \mathrm{AU}$ is the same as that of Class I YSOs, i.e. $\sim 32 \%$ (Connelley et al. 2008), the probability of drawing 2 Class 0 binary systems in this separation range is only $\sim 9 \%$. Similarly, the probability of drawing 14 single Class 0 protostars in the separation range 150-550 AU, assuming the binary fraction of Class I YSOs, i.e. $\sim 18 \%$ (Connelley et al. 2008), is even lower: $\sim 6 \%$. Therefore, we see that combining our sample with that of Looney et al. (2000) allows us to strengthen the trend pointed out at the beginning of this section, namely that the Class 0 binary fraction may be lower than that of Class I YSOs, at least on scales $\sim 150-550 \mathrm{AU}$. Nevertheless, the trend is only present at the $\$ 1.9 \sigma$ confidence level in the enlarged sample, and thus remains only marginally significant. Clearly, more interferometric observations of Class 0 objects taken at comparable angular resolutions would be needed to confirm this trend and firmly establish that binary properties evolve between the Class 0 and the Class I stage.

\subsection{Comparison with numerical models of binary fragmentation}

In this section, we compare the results of our high-resolution PdBI observations with the predictions of three published numerical models of star formation, in terms of multiplicity and spatial structure. The first model (Stamatellos \& Whitworth 2009) deals with the fragmentation of a massive disk around an already formed YSO of comparable mass. The second hydrodynamic simulation (Bate 2009) describes the collapse and fragmentation of a $50 M_{\odot}$ cluster-forming clump, and takes into account radiative feedback from formed protostellar objects. The third model (Hennebelle \& Fromang 2008; Hennebelle \& Ciardi 2009) includes the effect of magnetic fields and simulates the collapse of an individual cloud core into a protostellar system.

In order to compare the typical outcomes of these simulations with our PdBI observations, the model column density images $\left(\right.$ in $\mathrm{g} \mathrm{cm}^{-2}$ ) were put to the distance and declination of the Taurus and Perseus clouds, then converted into flux density maps (in $\mathrm{mJy} /$ beam) assuming optically thin dust emission at $1.3 \mathrm{~mm}$, and $\kappa_{1.3 \mathrm{~mm}}=0.01 \mathrm{~cm}^{2} \mathrm{~g}^{-1}$ (Ossenkopf \& Henning 1994) and $T_{\mathrm{d}}=10 \mathrm{~K}$ for the dust properties. The resulting maps were convolved with the typical uv-coverage of the PdBI in A configuration to produce the synthetic $1.3 \mathrm{~mm}$ continuum images presented in Figs. 8-10 below.

\subsubsection{Numerical simulations without magnetic fields}

\section{Disk fragmentation model}

The hydrodynamic simulations of Stamatellos \& Whitworth (2009), performed with the SPH code DRAGON, demonstrate that the outer parts of massive extended disks are likely to undergo gravitational fragmentation, thus forming low-mass companions. Because they fragment rapidly, such massive disks are unlikely to be observable beyond the Class 0 phase. We stress that protostellar collapse is not modeled in these simulations which have no protostellar envelope component.

Figure 8 a shows the model column density image resulting from an hydrodynamic simulation of a $0.7 M_{\odot}$ disk around a $0.7 M_{\odot}$ star, as seen $\sim 4500$ years after the start of the simulation (see Fig. 1 of Stamatellos \& Whitworth 2009 for more details). Figures $8 \mathrm{~b}$ and $8 \mathrm{c}$ present synthetic $1.3 \mathrm{~mm}$ continuum images resulting from simulations of $\mathrm{A}$-array $\mathrm{PdB}$ observations of the model placed at the distance of Taurus and Perseus, respectively. One can see from the dashed contour and first plain contour (corresponding to the average $3 \sigma$ and $5 \sigma$ levels achieved in our PdBI observations, respectively) that we expect the massive, extended disk of the model to be detected as a strong, well-resolved structure in IRAM PdBI observations. A circular Gaussian fit to the visibilities of the synthetic images shown in Figs. $8 \mathrm{~b}$ and $8 \mathrm{c}$ leads to a $F W H M$ diameter of $\sim 3.8^{\prime \prime} \pm 0.7^{\prime \prime}$ at the Taurus distance, and $\sim 1.8^{\prime \prime} \pm 0.4^{\prime \prime}$ at the Perseus distance: this is one order of magnitude greater than the FWHM computed in the same way for the 5 target sources detected in our PdBI maps (see Table 3), 


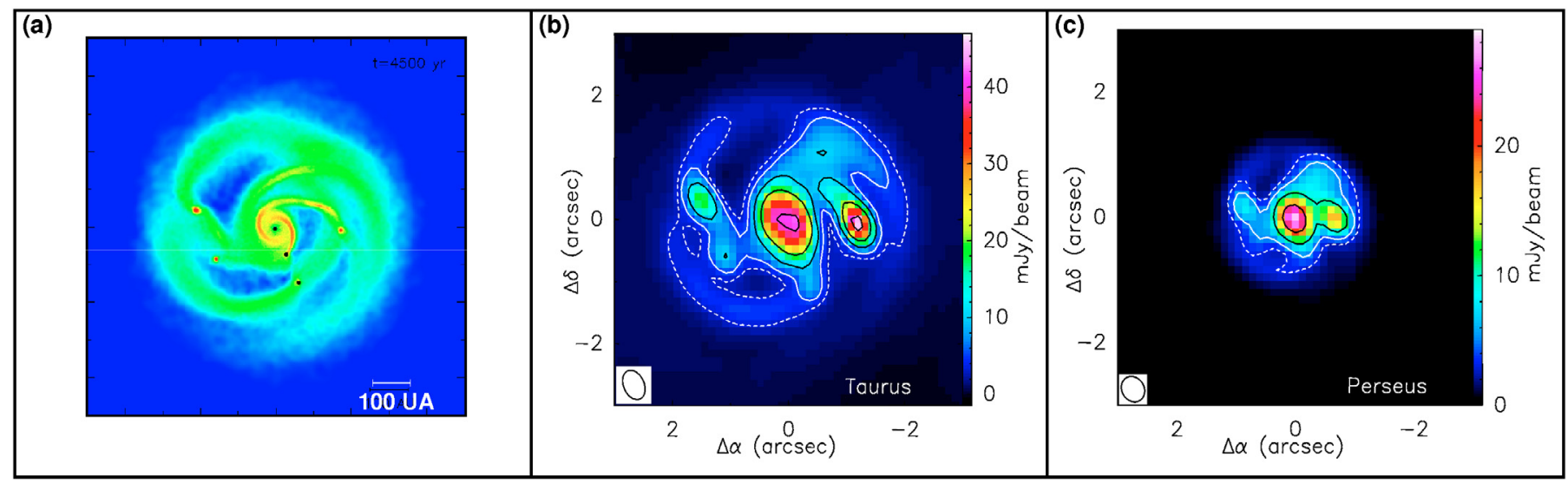

Fig. 8. a) Model column density image from the radiative hydrodynamic simulation of Stamatellos \& Whitworth (2009) (see text for further details). b) synthetic $1.3 \mathrm{~mm}$ continuum image resulting from simulated A-array PdB observations of the model shown in a), assuming a distance $d=140 \mathrm{pc}$ (distance to the Taurus complex). c) Same as b), but assuming a distance of $d=250 \mathrm{pc}$ (distance to the Perseus complex). The color scale is linear and gives an indicative flux density scale in mJy/beam. The peak flux density is found to be $\sim 46 \mathrm{mJy} / \mathrm{beam}$ and $\sim 29 \mathrm{mJy} / \mathrm{beam}$ in $\mathbf{b})$ and c), respectively. In both panels, the white dashed contour is showing the typical $3 \sigma$ detection level ( $3 \mathrm{mJy} / \mathrm{beam})$ achieved in our PdB-A observations, while the first plain contour is the typical $5 \sigma$ level. The following black contours are levels of 10,20 and $40 \sigma$. The ellipse in the bottom-left corner represents the beam size of PdB-A at the corresponding declination.
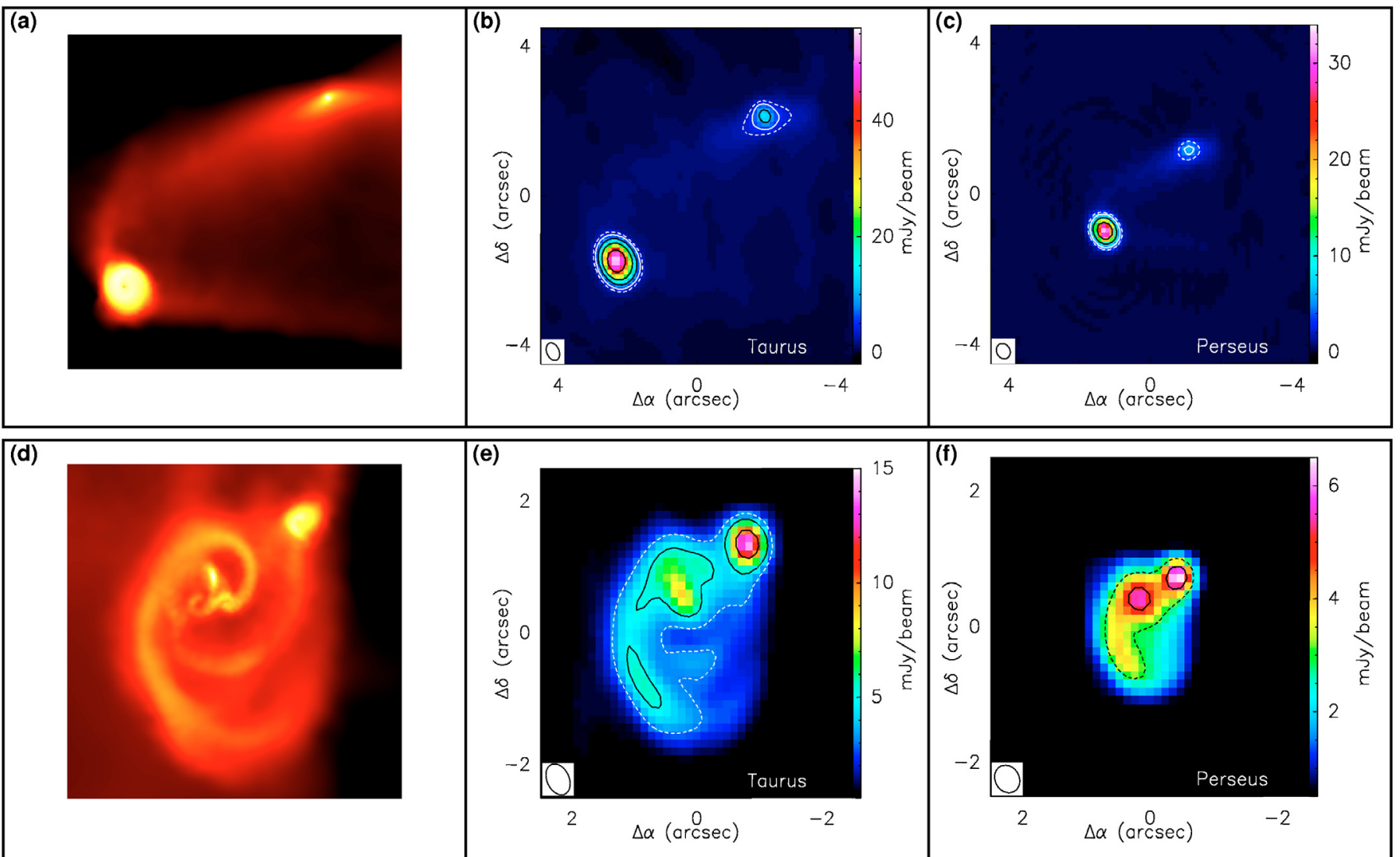

Fig. 9. Model column density images and synthetic $1.3 \mathrm{~mm}$ continuum images resulting from simulated A-array PdB observations of two typical outcomes from the model by Bate (2009) (seen $2.6 \times 10^{5}$ years after the start of the collapse, i.e 1.4 times the initial free fall time of the cloud). In each row the first image is the model column density image, while the two images on the right are the synthetic images obtained after convolution with the PdBI A-array configuration. a) is a $\sim 1000 \mathrm{AU}$ wide snapshot, while d) is $\sim 600 \mathrm{AU}$ wide. b) and e) were produced assuming distances $d=140 \mathrm{pc}$ (similar to the distance of the Taurus complex). c) and f) were produced assuming $d=250 \mathrm{pc}$ (similar to the distance of the L1448 complex). In all panels, the contours are levels of 3, 5, 10, 20 and $40 \sigma$, as achieved in our observations. The color scale is linear and gives an indicative flux density scale in mJy/beam. The dashed contour is showing the typical $3 \sigma$ detection level $(\sim 3 \mathrm{mJy} / \mathrm{beam})$ achieved in our PdB-A observations. The ellipse in the bottom-left corner represents the beam size of PdB-A at the corresponding declination. 

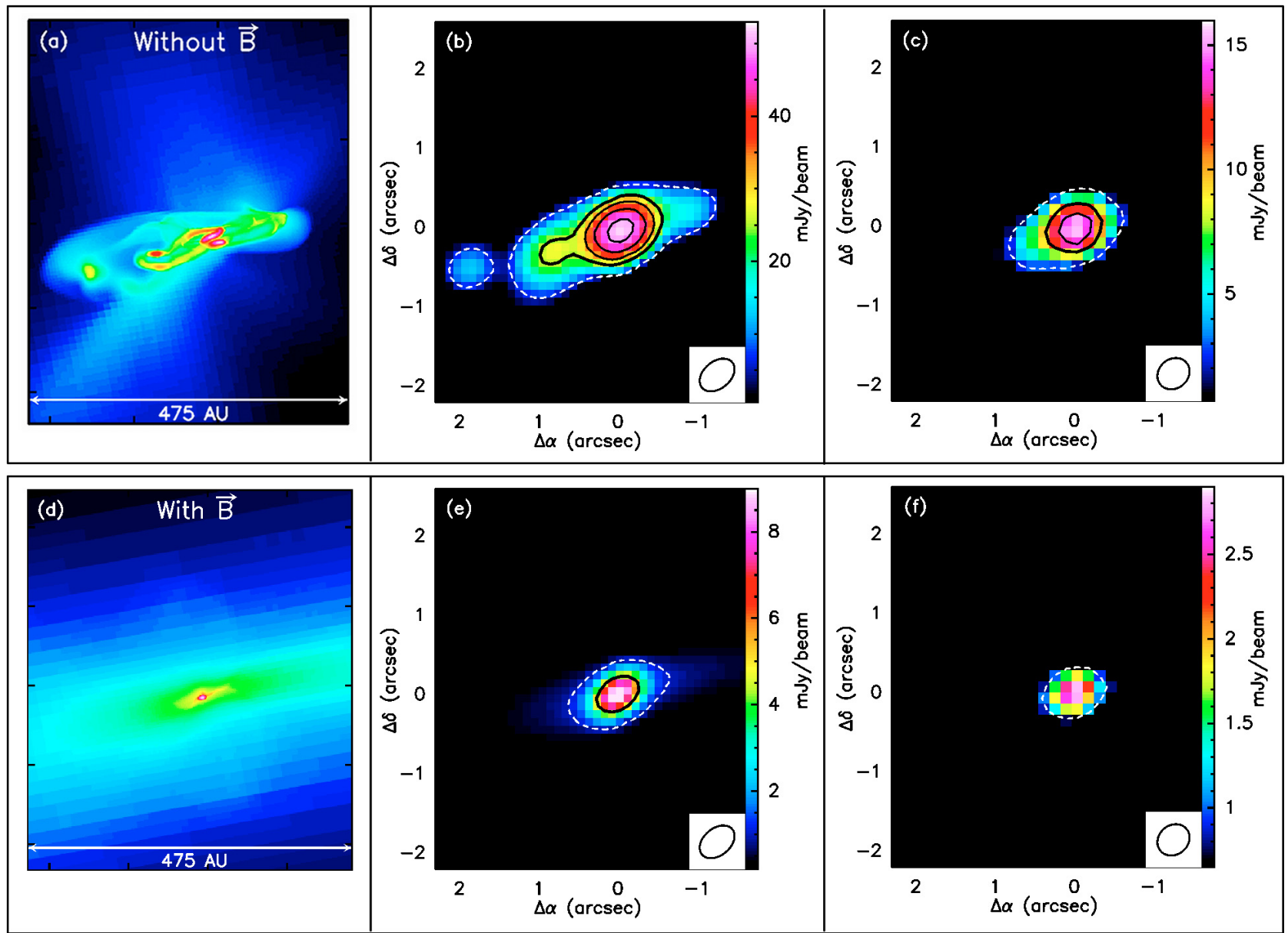

Fig. 10. Synthetic $1.3 \mathrm{~mm}$ continuum images resulting from simulated A-array PdB observations of two typical outcomes from the simulations of magnetized core collapse from e.g. Hennebelle \& Teyssier (2008) (see text for further details). Upper row: panel a) shows a model snapshot view of the column density distribution of the inner part of a protostellar system obtained $\sim 10^{4} \mathrm{yr}$ after the beginning of collapse in purely hydrodynamic $(B=0)$ simulations of cloud core collapse. Panel b) shows the synthetic PdB-A $1.3 \mathrm{~mm}$ dust continuum image produced from the purely hydrodynamic model a), put to the distance of the Taurus complex ( $d=140 \mathrm{pc}$ ). Panel c) shows a synthetic image similar to b), but put to the distance of the Perseus complex $(d=250 \mathrm{pc})$. Lower row: panel d) shows a snapshot view of the column density distribution of the inner part of a protostellar system obtained from MHD simulations starting from the same initial conditions as in panel a), except for a non-zero magnetic field (whose initial value is $1 / 2$ of the critical field strength required to prevent collapse). Panel e) shows the synthetic PdB-A $1.3 \mathrm{~mm}$ dust continuum image produced from the MHD model shown in d), and put to the distance of the Taurus complex $(d=140 \mathrm{pc})$. Panel f) shows a synthetic image similar to e), but put to the distance of the Perseus complex ( $d=250 \mathrm{pc}$ ). In all of the synthetic images, the color scale is linear and gives an indicative flux density scale in mJy/beam, assuming $T_{\mathrm{d}}=10 \mathrm{~K}$ and $\kappa_{1.3 \mathrm{~mm}}=0.01 \mathrm{~cm}^{2} \mathrm{~g}^{-1}$ for the dust properties. The white dashed contour shows the typical $3 \sigma$ detection level $(\sim 3 \mathrm{mJy} /$ beam) achieved in our PdB-A observations, while the following black contours are: $5 \sigma$ (thick), $10 \sigma, 20 \sigma$ and $40 \sigma$.

which show that all 5 target sources are compact when observed with the A array. Furthermore, two of the Taurus sources we observed (IRAM 04191 and L1521-F) have peak fluxes which are more than one order of magnitude weaker than the peak flux in the synthetic image of Fig. $8 \mathrm{~b}$.

Taken at face value, therefore, our PdBI results are not consistent with the model of Stamatellos \& Whitworth (2009). Note, however, that somewhat less massive $\left(\sim 0.1 M_{\odot}\right)$ disks, or initially massive disks observed at a later evolutionary stage, could be seen as compact structures, at the sensitivity achieved in our PdBI observations. One possible explanation for the absence of massive extended disks in our observations may be that the massive disks of the model are short-lived $\left(\sim 10^{4} \mathrm{yr}\right)$, as pointed out by Stamatellos \& Whitworth (2009). On the other hand, the presence of massive, infalling envelopes around the Class $0 \mathrm{ob}-$ jects we observed (which are not modeled in the simulations by
Stamatellos \& Whitworth 2009) should tend to refill any extended disk present at early times. A more likely explanation in the context of this model is that only $\sim 20-30 \%$ of all solartype protostars may develop massive extended disks at any time in their evolution (Stamatellos \& Whitworth 2009). Our present sample is clearly not large enough to rule out this possibility.

Therefore, while current numerical simulations of massive, extended disks do not satisfactorily reproduce our PdBI observations, more numerical simulations and more high-resolution observations of Class 0 objects would be needed before robust conclusions can be drawn on this scenario.

Hydrodynamic model including cloud collapse, disk formation, and radiative feedback

In recent hydrodynamical simulations, Bate (2009) treats both cloud collapse and protostar/disk formation, including the effect of radiative feedback from newly formed protostellar objects. 
The two model snapshots presented in Figs. 9a and 9d (see also Fig. 4 of Bate 2009) were processed through the PdB simulator to produce the synthetic A-array $1.3 \mathrm{~mm}$ continuum images shown in Figs. 9b, e and 9c, f, with the models placed at the distances of Taurus and Perseus, respectively. One can see from the dashed level and first plain level (corresponding to the average $3 \sigma$ and $5 \sigma$ levels achieved in our PdBI observations, respectively) that the large disk-like rotating structures produced by the models are expected to be detected as strong, extended or multiple sources in A-array PdBI observations. A circular Gaussian fit to the visibilities of the synthetic images shown in Figs. 9e and 9f leads to a FWHM diameter of $\sim 3.4^{\prime \prime} \pm 0.7^{\prime \prime}$ at the Taurus distance, and $\sim 1.9^{\prime \prime} \pm 0.5^{\prime \prime}$ at the Perseus distance: this is again one order of magnitude greater than the FWHM diameter measured in the same way for the 5 target sources detected in our PdBI maps (see Table 3), which show that all 5 target sources are compact when observed with the A array.

We conclude that purely hydrodynamic simulations, even if they include radiative feedback which inhibits fragmentation close to existing protostellar objects, fail to reproduce our PdBI observations, because they tend to form massive extended structures (with $F W H M \sim 300-500 \mathrm{AU}$ ) and/or multiple systems. One should bear in mind, however, that the absolute age of Class 0 objects $\left(\sim 3 \times 10^{4}-2 \times 10^{5} \mathrm{yr}\right)$ is quite uncertain (cf. Evans et al. 2009), which casts some doubt on the time at which the models should be compared to our observations.

\subsubsection{Magnetohydrodynamic model}

Adaptive Mesh Refinement (AMR) simulations of cloud core collapse and fragmentation were carried out by Hennebelle \& Fromang (2008) and Hennebelle \& Teyssier (2008), using the MHD version of the RAMSES code (see Fromang et al. 2006). In these MHD simulations, the formation of a centrifugallysupported disk is suppressed by magnetic braking (see Mellon \& Li 2008). Moreover, Hennebelle \& Teyssier (2008) showed that, for rotating dense cores with a magnetic field strength typical of values inferred from observations (e.g. Crutcher 1999), fragmentation was suppressed by magnetic fields if the initial density perturbations were too small.

Two typical outcomes of these simulations are shown in Fig. 10: Figs. 10a and 10d are model column density images obtained in the absence and in the presence of a magnetic field, respectively. Figures $10 \mathrm{~b}$ and $10 \mathrm{e}$ represent synthetic images obtained from these models after convolution with the dirty beam of PdBI in A configuration and cleaning, assuming a distance $d=140 \mathrm{pc}$, while Figs. 10c and 10f represent the same synthetic images, but assuming a distance $d=250 \mathrm{pc}$. The simulations shown in the upper and lower panels started from a rotating, centrally-condensed spherical core 0.013 pc in radius, with a density contrast of 10 . The total mass of the core was $2 M_{\odot}$, and the two simulations started from the same initial conditions, except for the initial value of the magnetic field $(B=0$ in the first case; moderate B-field strength in the second case, i.e., initial mass-to-flux ratio $\mu=3.2$ in units of the critical value for collapse). Note that the purely hydrodynamic simulation of Fig. 10a produces a quadruple system within a radius of $\sim 200 \mathrm{AU}$, while core fragmentation is completely suppressed in the moderately magnetized simulation of Fig. 10d, which leads to the formation of a single stellar object within $200 \mathrm{AU}$.

The magnetized model shown in the lower panels of Fig. 10 reproduces our PdBI observations of the Taurus Class 0 protostars quite well: the synthetic image (Fig. 10e) shows a unique source, with a peak flux density $\sim 3-8 \mathrm{mJy} /$ beam, and a size comparable to those observed: a circular Gaussian fit to the visibilities of the synthetic images shown in Figs. 10e leads to a FWHM diameter of $\sim 0.4^{\prime \prime}$ at the Taurus distance $\left(\sim 0.2^{\prime \prime}\right.$ at the Perseus distance), comparable to the ones computed for the sources detected in our PdBI maps, and given in Table 3. This comparison suggests that magnetic fields are an essential ingredient of the early phases of star formation, as they seem to be a plausible way to regulate core/disk fragmentation and obtain single objects on scales $<300 \mathrm{AU}$, similar to what is observed in our sample.

\subsubsection{Implications for the formation of multiple systems}

Although our sample clearly needs to be extended before general conclusions can be drawn, the non-detection of multiple Class 0 systems with separations between $\sim 100$ AU and $\$ 600$ AU in both our sample and the BIMA sample of Looney et al. (2000) already suggests interesting implications for the formation of solar-type multiple systems.

Two alternative scenarios can be proposed. First, it is possible that wide (>600 AU) multiple systems form during the Class 0 phase, and that orbital migration then reduces the separation between the protostellar components during evolution to the Class I phase (Bate 2000; Bate et al. 2002). Observations (see, for example Connelley et al. 2008) suggest that main-sequence systems are tighter than T Tauri systems, and that the latter themselves have separations which are somewhat smaller than those observed toward Class I YSOs (Patience et al. 2002). Therefore, it is possible that the typical separation of multiple systems decreases in the course of YSO evolution (Connelley et al. 2008), even if such an effect is not yet well understood. The formation of wide binary systems at the Class 0 stage has been envisaged by Price \& Bate (2007) and Hennebelle \& Teyssier (2008). Based on their MHD numerical simulations, these authors showed that, in the presence of large-amplitude initial perturbations, each perturbation develops independently leading to the formation of a wide protobinary system, which can then gravitationally contract to typical PMS binary separations.

A second possible scenario would be that multiple systems form with tight separations $\leq 75 \mathrm{AU}$, explaining the paucity of systems in the $\sim 100-500$ AU separation range at the Class 0 stage, but that these systems then expand to produce multiple systems with wider typical separations at the Class I stage. This alternative scenario is plausible if fragmentation occurs during the second collapse phase, a possibility which has been explored in the numerical simulations of Bonnell (1994) and Machida et al. (2007). One of the conditions for this model to produce binary systems with typical separations $\sim 100-500$ AU is that the initially very tight protobinary system must gain sufficient angular momentum by accretion (see e.g. Goodwin et al. 2004; Bate 2000) to increase the separation between the two protostellar components by the end of the Class 0 phase.

\section{Summary and conclusions}

We carried out a subarcsecond pilot study of 5 Class 0 objects at $1.3 \mathrm{~mm}$ with the IRAM Plateau de Bure Interferometer in its most extended configuration, in an effort to probe protostellar multiplicity at separations $50<a<5000 \mathrm{AU}$ at the beginning of the embedded YSO phase. Continuum emission and ${ }^{12} \mathrm{CO}(2-1)$ emission were observed simultaneously, with a typical resolution $\sim 0.3^{\prime \prime}-0.5^{\prime \prime}$ and rms sensitivity $\sim 0.1-1 \mathrm{mJy} /$ beam, which allowed us to study multiplicity down to separations $a \sim 50 \mathrm{AU}$ and circumstellar mass ratios $q \sim 0.07$. 

follows:

Our main results and conclusions can be summarized as

1. All five primary Class 0 protostars (IRAM 04191, L1527, L1521-F, L1448-C, and NGC1333-IR2A) were detected in the $1.3 \mathrm{~mm}$ continuum maps, with signal-to-noise ratios ranging from 5.4 to 65.5 .

2. Toward L1448-C, a series of seven high-velocity ( $v_{\text {LSR }}>$ $50 \mathrm{~km} \mathrm{~s}^{-1}$ ) bullets were detected in ${ }^{12} \mathrm{CO}(2-1)$, which trace the axis of the bipolar jet in both the redshifted and blueshifted lobes of the outflow.

3. Single $1.3 \mathrm{~mm}$ continuum components associated with the primary Class 0 objects were detected in the case of the three Taurus sources, while robust evidence of secondary components was found toward the two Perseus sources, L1448-C and NGC1333-IR2A.

4. The L1448-C secondary component lies 600 AU south-east of the primary source, at a position angle close to that of the $\mathrm{CO}(2-1)$ jet axis. We show that it is not a genuine protostellar companion but rather an outflow feature directly associated with the powerful jet driven by L1448-C. The detection of compact millimeter continuum emission originating from such an outflow-generated feature emphasizes the need to observe outflow-shock diagnostics, before any robust conclusion can be drawn on the nature of secondary components detected in the vicinity of protostellar objects.

5. The nature of the NGC 1333-IR2A secondary component, detected $\sim 1900$ AU south-east of the primary source, is as yet unclear: it may either be a genuine protostellar companion or trace the edge of an outflow cavity.

6. Altogether, our pilot PdBI survey found only evidence of outflow-generated features, and/or wide protobinary systems: no multiple system was detected at separations $a<$ $1900 \mathrm{AU}$ in our sample of 5 Class 0 protostars.

7. Combining our results with the BIMA survey of 9 Class 0 objects by Looney et al. (2000), we argue that there is no evidence of multiple protostellar systems in the separation range $150<a<550$ AU among an enlarged sample of 14 Class 0 protostars. Although the millimeter interferometric observations available for this enlarged sample are inhomogeneous, they tentatively suggest that the Class 0 binary fraction may be lower than that of Class I YSOs, at least for separations 150-500 AU. This tentative evolution of the binary fraction from the Class 0 to the Class I stage is present at the $\sim 1.9 \sigma$ confidence level in the enlarged sample, and thus requires confirmation.

8. Comparison of synthetic model images with our PdBI results shows that purely hydrodynamic models of protostellar collapse and disk formation have difficulties matching our observations, since these models typically produce multiple components, embedded in large-scale rotating structures, which are not observed toward our sample of five Class 0 sources. These large-scale rotating structures may be shortlived, however, and more observations would be needed to draw robust conclusions, given the currently large uncertainties on the Class 0 lifetime.

9. Comparison of synthetic model images from magnetohydrodynamic models with our PdBI results shows that magnetized models of protostar formation agree better with our observations, as magnetic fields tend to prevent the formation of extended disk-like structures and to suppress fragmentation into multiple components on small scales (100-1000 AU).
10. However, magnetohydrodynamic models may allow wide $(\geq 1000 \mathrm{AU})$ and/or very tight ( $\leq 30 \mathrm{AU})$ multiple systems to form during the Class 0 phase. The paucity of multiple Class 0 systems with separations $150 \leq a \leq 600 \mathrm{AU}$, if confirmed by comparable observations of larger source samples, may thus favor binary formation scenarios which involve dynamical evolution of the system separations with time.

Acknowledgements. We are grateful to the IRAM-PdBI staff, and more specifically to Roberto Neri and Frédéric Gueth, for their precious help with the PdBI observations and data processing. We also thank Mario Tafalla for helpful input during the preparatory phase of this project. The work presented in this paper was stimulated by discussions held in the context of the Marie Curie Research Training Network "Constellation" (MRTN-CT2006- 035890).

\section{References}

André, P., \& Montmerle, T. 1994, ApJ, 420, 837

André, P., Motte, F., \& Bacmann, A. 1999, ApJ, 513, L57

André, P., Ward-Thompson, D., \& Barsony, M. 1993, ApJ, 406, 122

André, P., Ward-Thompson, D., \& Barsony, M. 2000, Protostars and Planets IV, 59

Bachiller, R., Martin-Pintado, J., Tafalla, M., Cernicharo, J., \& Lazareff, B. 1990, A\&A, 231, 174

Bachiller, R., André, P., \& Cabrit, S. 1991, A\&A, 241, L43

Bachiller, R., Guilloteau, S., Dutrey, A., Planesas, P., \& Martin-Pintado, J. 1995, A\&A, 299, 857

Barsony, M., Ward-Thompson, D., André, P., \& O'Linger, J. 1998, ApJ, 509, 733

Bate, M. 2000, MNRAS, 314, 33

Bate, M. 2009, MNRAS, 392, 1363

Bate, M., Bonnell, I., \& Bromm, V. 2002, MNRAS, 332, L65

Belloche, A., André, P., Despois, D., \& Blinder, S. 2002, A\&A, 393, 927

Bonnell, I. A. 1994, MNRAS, 269, 837

Bonnell, I. A., \& Bate, M. R. 1994, MNRAS, 271, 999

Bontemps, S., \& André, P. 1997, in Herbig-Haro Flows and the Birth of Stars, ed. B. Reipurth, \& C. Bertout, IAU Symp., 182, 63

Bourke, T. L. 2001, ApJ, 554, L91

Bourke, T. L., Myers, P. C., Evans, II, N. J., et al. 2006, ApJ, 649, L37

Chabrier, G. 2005, in The Initial Mass Function 50 Years Later, ed. E. Corbelli, F. Palla, \& H. Zinnecker, Ap\&SS Library, 327, 41

Chandler, C., \& Richer, J. 2000, ApJ, 530, 851

Chen, H., Myers, P., Ladd, E., \& Wood, D. 1995, ApJ, 445, 377

Chen, X., Launhardt, R., Bourke, T. L., Henning, T., \& Barnes, P. J. 2008, ApJ, 683,862

Clark, B. 1980, A\&A, 89, 377

Codella, C., Welser, R., Henkel, C., Benson, P. J., \& Myers, P. C. 1997, A\&A, 324, 203

Commerçon, B., Hennebelle, P., Audit, E., Chabrier, G., \& Teyssier, R. 2008, A\&A, 482, 371

Connelley, M. S., Reipurth, B., \& Tokunaga, A. T. 2008, AJ, 135, 2526

Crapsi, A., Caselli, P., Walmsley, C. M., et al. 2004, A\&A, 420, 957

Curiel, S., Raymond, J. C., Moran, J. M., Rodriguez, L. F., \& Canto, J. 1990, ApJ, 365, L85

Curiel, S., Torrelles, J. M., Rodríguez, L. F., Gómez, J. F., \& Anglada, G. 1999, ApJ, 527, 310

Dent, W. R. F., Matthews, H. E., \& Ward-Thompson, D. 1998, MNRAS, 301, 1049

Dionatos, O., Nisini, B., Garcia Lopez, R., et al. 2009, ApJ, 692, 1

Duchêne, G., Bouvier, J., Bontemps, S., André, P., \& Motte, F. 2004, A\&A, 427, 651

Duchêne, G., Bontemps, S., Bouvier, J., et al. 2007, A\&A, 476, 229

Dunham, M. M., Evans, II, N. J., Bourke, T. L., et al. 2006, ApJ, 651, 945

Enoch, M. L., Young, K. E., Glenn, J., et al. 2006, ApJ, 638, 293

Evans, N. J., Dunham, M. M., Jørgensen, J. K., et al. 2009, ApJS, 181, 321

Fromang, S., Hennebelle, P., \& Teyssier, R. 2006, A\&A, 457, 371

Fuller, G. A., Ladd, E. F., Padman, R., Myers, P. C., \& Adams, F. C. 1995, ApJ, 454,862

Ghavamian, P., \& Hartigan, P. 1998, ApJ, 501, 687

Ghez, A. M., Neugebauer, G., \& Matthews, K. 1993, AJ, 106, 2005

Girart, J. M., Rao, R., \& Estalella, R. 2009, ApJ, 694, 56

Goodwin, S. P., Kroupa, P., Goodman, A., \& Burkert, A. 2007, in Protostars and Planets V, ed. B. Reipurth, D. Jewitt, \& K. Keil, 133

Goodwin, S. P., Whitworth, A. P., \& Ward-Thompson, D. 2004, A\&A, 423, 169 Greene, T., Wilking, B., Andre, P., Young, E., \& Lada, C. 1994, ApJ, 434, 614 Guilloteau, S., Bachiller, R., Fuente, A., \& Lucas, R. 1992, A\&A, 265, L49 
A. J. Maury et al.: Toward understanding the formation of multiple systems

Hartigan, P. 2003, Ap\&SS, 287, 111

Hennebelle, P., \& Ciardi, A. 2009, A\&A, 506, L29

Hennebelle, P., \& Fromang, S. 2008, A\&A, 477, 9

Hennebelle, P., \& Teyssier, R. 2008, A\&A, 477, 25

Hennebelle, P., Whitworth, A., Cha, S.-H., \& Goodwin, S. 2004, MNRAS, 348, 687

Hogerheijde, M., van Dishoeck, E., Blake, G., \& van Langevelde, H. 1998, ApJ, 502,315

Jørgensen, J. K., Hogerheijde, M. R., van Dishoeck, E. F., Blake, G. A., \& Schöier, F. L. 2004, A\&A, 413, 993

Jørgensen, J. K., Harvey, P. M., Evans, II, N. J., et al. 2006, ApJ, 645, 1246

Jørgensen, J. K., Bourke, T. L., Myers, P. C., et al. 2007, ApJ, 659, 479

Knee, L. B. G., \& Sandell, G. 2000, A\&A, 361, 671

Köhler, R., Neuhäuser, R., Krämer, S., et al. 2008, A\&A, 488, 997

Kroupa, P. 2001, MNRAS, 322, 231

Lada, C. J. 2006, ApJ, 640, L63

Ladd, E. F., Adams, F. C., Fuller, G. A., et al. 1991, ApJ, 382, 555

Lefloch, B., Cernicharo, J., Cabrit, S., \& Cesarsky, D. 2005, A\&A, 433, 217

Liseau, R., Sandell, G., \& Knee, L. B. G. 1988, A\&A, 192, 153

Looney, L., Mundy, L., \& Welch, W. 2000, ApJ, 529, 477

Machida, M. N., Matsumoto, T., Hanawa, T., \& Tomisaka, K. 2005, MNRAS, 362,382

Machida, M. N., Inutsuka, S.-i., \& Matsumoto, T. 2007, ApJ, 670, 1198
Machida, M. N., Tomisaka, K., Matsumoto, T., \& Inutsuka, S.-i. 2008, ApJ, 677, 327

Mellon, R. R., \& Li, Z.-Y. 2008, ApJ, 681, 1356

Motte, F., \& André, P. 2001, A\&A, 365, 440

Ohashi, N., Hayashi, M., Ho, P., \& Momose, M. 1997, ApJ, 475, 211

Onishi, T., Mizuno, A., \& Fukui, Y. 1999, PASJ, 51, 257

Ossenkopf, V., \& Henning, T. 1994, A\&A, 291, 943

Parker, N., Padman, R., \& Scott, P. 1991, MNRAS, 252, 442

Patience, J., Ghez, A. M., Reid, I. N., \& Matthews, K. 2002, AJ, 123, 1570

Price, D. J., \& Bate, M. R. 2007, MNRAS, 377, 77

Reipurth, B., \& Clarke, C. 2001, AJ, 122, 432

Reipurth, B., Rodríguez, L., Anglada, G., \& Bally, J. 2002, AJ, 124, 1045

Robitaille, T. P., Whitney, B. A., Indebetouw, R., \& Wood, K. 2007, ApJS, 169, 328

Rodríguez, L., Anglada, G., \& Curiel, S. 1999, ApJS, 125, 427

Sandell, G., \& Knee, L. B. G. 2001, ApJ, 546, L49

Sandell, G., Knee, L. B. G., Aspin, C., Robson, I. E., \& Russell, A. P. G. 1994, A\&A, 285, L1

Simon, M., Ghez, A., Leinert, C., et al. 1995, ApJ, 443, 625

Stamatellos, D., \& Whitworth, A. 2009, MNRAS, 392, 413

Terebey, S., Fich, M., Noriega-Crespo, A., et al. 2009, ApJ, 696, 1918

Tohline, J. E. 2002, ARA\&A, 40, 349

Woitas, J., Leinert, C., \& Köhler, R. 2001, A\&A, 376, 982 